\title{
Compatible abelian symmetries in N-Higgs-doublet models
}

\section{C.C. Nishi}

Universidade Federal do $A B C-U F A B C$, 09.210-170, Santo André, SP, Brazil

Maryland Center for Fundamental Physics, University of Maryland, College Park, MD 20742, U.S.A.

E-mail: celso.nishi@ufabc.edu.br

ABSTRACT: We analyze the compatibility between abelian symmetries acting in two different sectors of a theory using the Smith Normal Form method. We focus on N-Higgs-doublet models (NHDMs) and on the compatibility between symmetries in the Higgs potential and in the Yukawa interactions, which were separately analyzed in previous works. It is shown that two equal (isomorphic) symmetry groups that act in two separate sectors are not necessarily compatible in the whole theory and an upper bound is found for the size of the group that can be implemented in the entire NHDM. We also develop useful techniques to analyze compatibility and extend a symmetry from one sector to another. Consequences to the supersymmetric case are briefly discussed.

KEywords: Discrete and Finite Symmetries, Beyond Standard Model

ARXiv EPRINT: 1411.4909 


\section{Contents}

1 Introduction $\quad 2$

2 Review of the method 3

2.1 Example 3

2.2 Charges are not unique 5

2.3 Notation 6

$\begin{array}{lll}2.4 & \text { Linear independence } & 7\end{array}$

2.5 Height of vector 8

3 Matching up symmetries $\quad 8$

4 Maximal symmetries for three Higgs doublets 11

$\begin{array}{lll}4.1 & \mathbb{Z}_{4}-3 \mathrm{HDM} & 14\end{array}$

$4.2 \mathbb{Z}_{2} \times \mathbb{Z}_{2}-3 \mathrm{HDM} \quad 14$

5 Discarding maximal symmetries $\quad \mathbf{1 4}$

5.1 Discarding maximal symmetries for $N=4$ and $N=5 \quad 15$

5.2 Discarding maximal symmetries for $N \geq 6 \quad 16$

6 Building symmetric models $\quad 18$

6.1 From minimal to maximal number of terms 18

$\begin{array}{ll}\text { 6.2 From the Yukawa sector to the potential } & 19\end{array}$

6.3 From the potential to the Yukawa sector 19

6.4 From reduced $D^{Y}$ to full $D^{Y} \quad 20$

$\begin{array}{lll}7 & \text { Discussion and conclusion } & 23\end{array}$

$\begin{array}{ll}\text { A Proof of proposition } & 25\end{array}$

B Rephasing space $\quad 25$

C Backbone structure for maximal symmetry in 3HDM 26

$\begin{array}{ll}\text { D Height of } \mathrm{p}_{i}^{\prime} \text { vectors for maximal } D^{Y} & 29\end{array}$

$\mathrm{E}$ Height of $\mathrm{p}_{3}^{\prime}$ can not be reduced $\quad 30$

$\begin{array}{lll}\text { F } & \text { Examples of full models and textures } & 31\end{array}$ 


\section{Introduction}

Symmetry has always played a crucial role in our understanding of fundamental physics. The construction of the current framework - the Standard Model (SM) of particle physics - has culminated in 2012 with the discovery of the Higgs boson [1, 2], the particle that results from the breaking of the electroweak symmetry in its simplest form. Hence, it was also a successful attempt to probe a hidden (broken) symmetry in nature and its breaking mechanism. However, as we probe higher and higher energies, new symmetries may emerge as key ingredients to understand the physics beyond the SM.

As we try to guess which new symmetry governs the physics above the electroweak scale, we are also confronted with the question of what is the breaking scale and what could be the signatures after breaking. One old but fruitful example where the symmetry should (usually) be broken at very high energies is $B-L$ symmetry, a symmetry that might be linked to the smallness of neutrino masses (see, e.g., ref. [3] and references therein).

In parallel to continuous symmetries, discrete symmetries are also possible ingredients with which we can understand flavor (for a review, see e.g. refs. [4-8]) and the stability of dark matter (with, e.g., R-parity [9] or matter parity [10]). In the effort to classify and discover useful abelian discrete symmetries, the Smith Normal Form (SNF) method has been used successfully in various contexts to find discrete symmetries arising from the breaking of continuous gauge symmetries [11, 12], find useful R-symmetries in supersymmetric extensions of the SM [13], justify two-zero textures in the neutrino mass matrix with symmetries [14] and classify abelian symmetries in multi-Higgs-doublets models [15]. The latter class of models will be the focus of this work.

The N-Higgs-doublet models (NHDMs) are among the most conservative extensions of the SM and they can present additional features that are absent in the single-Higgsdoublet SM such as spontaneous CP violation $[16,17]$ or geometric CP violation [18-21]. In many ways, these new phenomena are possible because the scalar potential has more structure to allow different symmetry breaking paths. Along with more structure comes the possibility of accommodating larger symmetries, specially discrete symmetries. One can for example impose a $\mathbb{Z}_{2}$ symmetry to naturally suppress dangerous flavor changing currents for quarks [22] or obtain a dark matter candidate with radiative neutrino mass generation [23]. The list of all possible symmetries that can be accommodated in the $2 \mathrm{HDM}$ is short and the groups in it are small [24-30] (more symmetries arise if we allow for accidental symmetries [31-36]). More and larger discrete symmetries are possible in the $3 \mathrm{HDM}$ potential [37], and all possible breaking patterns were summarized recently in ref. [38]. In general, we can accommodate larger symmetries as we add more fields. If we are restricted to abelian symmetries, the maximal order of the group that can be separately implemented in the Higgs potential and in the Yukawa interactions were presented in refs. [39] and [15], respectively.

In this work, we want to extend the methods of [15] to consider the compatibility issues, i.e., (i) how to analyze if a symmetry acting in two sectors of a theory are compatible and (ii) how to extend a given symmetry from one sector to another. As an example of a situation where we want an answer to (i), we know $\mathbb{Z}_{8}$ is the largest symmetry we can 
implement separately in the Higgs potential and in the Yukawa interactions for four Higgs doublets $[15,39]$ but it is not clear if the same symmetry can be valid for the entire theory. In contrast, the necessity for (ii) arises frequently when a symmetry is interesting in one sector of the theory and we want to build the entire theory. To answer these questions, we need to formulate compatibility conditions in terms of the Smith Normal form and develop techniques to check and extend symmetries.

The outline of this work is as follows: in section 2 we review the method of the Smith Normal Form to analyze discrete and continuous abelian symmetries. We formulate the compatibility conditions in the context of N-Higgs-doublet models in section 3, although they can be easily adapted to any theory with two sectors or more. Section 4 shows that the groups with maximal order in the potential can be extended compatibly to the whole theory for $N=2,3$. On the other hand, we show in section 5 that the groups of maximal order in the Yukawa sector, for $N \geq 4$, cannot be extended to the potential without leading to accidental symmetries. Methods to extend a symmetry from one sector to another are developed in section 6 and we conclude in section 7, where we also briefly discuss the supersymmetric case. Auxiliary material can be found in the appendices.

\section{Review of the method}

We review here the method to apply the Smith normal form (SNF) to analyze the rephasing symmetries of a Lagrangian composed of a polynomial of fields. More details can be found in ref. [15]. We just recall the definition of a realizable abelian symmetry: an abelian symmetry is realizable if there are no larger abelian symmetry containing it. Other approaches can be seen in refs. $[11,12]$ and in the references in the introduction. Note that Mathematica packages are available to promptly compute the SNF [40].

\subsection{Example}

Let us take as an example a Lagrangian depending on three scalar fields $\phi_{1}, \phi_{2}, \phi_{3}$, such as Higgs fields, appearing in the Lagrangian through only two phase sensitive terms,

$$
\phi_{2}^{\dagger} \phi_{1} \phi_{2}^{\dagger} \phi_{3}+\left(\phi_{3}^{\dagger} \phi_{1}\right)^{2} \subset \mathscr{L}
$$

We have suppressed the numerical coefficients.

The information on rephasing symmetries of the model is all encoded in the $D$ matrix [15],

$$
D=\left(\begin{array}{ccc}
1 & -2 & 1 \\
2 & 0 & -2
\end{array}\right),
$$

where each row corresponds to one phase sensitive term in the Lagrangian in (2.1) by applying the following rule: attribute one unit of a field specific charge to each field and list the cumulative charges of each field [we use the order $\left(\phi_{1}, \phi_{2}, \phi_{3}\right)$ in $(2.2)$ ].

The appearance of the integer $D$-matrix can be understood as follows [15]: apply general rephasing transformations

$$
\phi_{j} \rightarrow e^{i 2 \pi a_{j}} \phi_{j}
$$


Then the two terms in (2.1) gain phases $2 \pi\left(a_{1}-2 a_{2}+a_{3}\right)$ and $2 \pi\left(2 a_{1}-2 a_{3}\right)$, which should be integer multiples of $2 \pi$ to leave the Lagrangian invariant. In other words, the transformations (2.3) correspond to a rephasing symmetry if

$$
\begin{aligned}
a_{1}-2 a_{2}+a_{3}=b_{1} & \text { is integer } \\
2 a_{1}-2 a_{3}=b_{2} & \text { is integer } .
\end{aligned}
$$

In matricial notation, the condition is simply

$$
D A=B \quad \in \quad \mathbb{Z}^{2} \equiv \mathbb{Z} \times \mathbb{Z}
$$

where $A=\left(a_{1}, a_{2}, a_{3}\right)^{\top}$ and $B=\left(b_{1}, b_{2}\right)^{\top}$. However, not all solutions of (2.5) for $A$ are relevant: if all $a_{i}$ are integers, the solution is trivial. We are only interested in nontrivial solutions where $A \notin \mathbb{Z}^{3}$, which correspond to nontrivial discrete or continuous transformations of the type (2.3).

Deciding if eq. (2.5) possesses nontrivial solutions for $A$ is most easily accomplished if we can transform basis for $A, B$ (within integers) so that $D$ has diagonal form. Such a basis can be always found. By one elementary row operation and two column operations, the $D$-matrix can be transformed to the Smith Normal Form:

$$
\operatorname{SNF}(D)=\left(\begin{array}{lll}
1 & 0 & 0 \\
0 & 4 & 0
\end{array}\right) .
$$

If we apply the necessary row and column operations on the respective identity matrices, we obtain

$$
R=\left(\begin{array}{cc}
1 & 0 \\
-2 & 1
\end{array}\right), \quad C=\left(\begin{array}{lll}
1 & 2 & 1 \\
0 & 1 & 1 \\
0 & 0 & 1
\end{array}\right)
$$

which performs the diagonalization:

$$
R D C=\operatorname{SNF}(D)
$$

Hence, condition (2.5) can be rewritten as

$$
D^{\prime} A^{\prime}=B^{\prime} \in \mathbb{Z}^{2}
$$

where $D^{\prime}=\operatorname{SNF}(D), A^{\prime}=C^{-1} A$ and $B^{\prime}=R B$. Since $R, C$ are unimodular, they univocally map integer vectors into integer vectors and nontrivial solutions in the new basis correspond to nontrivial solutions in the original basis.

In the new basis, it easy to see that the only nontrivial solutions are $A^{\prime}=t(0,1,0)^{\top}$, with $t=1 / 4,2 / 4,3 / 4$, and $A^{\prime}=t(0,0,1)^{\top}$, with continuous $t$. In the original basis, they correspond to

$$
\begin{array}{ll}
A=t(2,1,0)^{\top}, & t=1 / 4,2 / 4,3 / 4, \\
A=t(1,1,1)^{\top}, & t \in \mathbb{R} .
\end{array}
$$


where the generating vectors correspond to the second and third columns of $C$, respectively. In terms of rephasing transformations (2.3), the solution in the first line of (2.10) corresponds to a discrete $\mathbb{Z}_{4}$ symmetry whereas the second solution corresponds to a continuous $\mathrm{U}(1)$ symmetry. They correspond to rephasing symmetries (2.3):

$$
\begin{aligned}
\mathbb{Z}_{4}:\left(\phi_{1}, \phi_{2}, \phi_{3}\right) & \sim\left(e^{i 2 \pi \frac{2 k}{4}}, e^{i 2 \pi \frac{k}{4}}, 1\right), \quad k=0,1,2, \\
\mathrm{U}(1):\left(\phi_{1}, \phi_{2}, \phi_{3}\right) & \sim\left(e^{i 2 \pi t}, e^{i 2 \pi t}, e^{i 2 \pi t}\right), \quad t \in \mathbb{R} .
\end{aligned}
$$

We can now see that all information on abelian symmetries, i.e., the symmetries and their generators, can be extracted from $\operatorname{SNF}(D)$ and $C$, respectively, without the need to perform all the basis change. We read these informations in the following way: the SNF form (2.6) is unique and implies that our symmetry is $\mathbb{Z}_{4} \times U(1)$, where $\mathbb{Z}_{4}$ is given by the nonzero and non-unit factor 4 in the diagonal and $\mathrm{U}(1)$ is characterized by the presence of one zero column. The U(1) symmetry is generated by the last column of $C$ in (2.7) since

$$
D \mathbf{s}=\mathbf{0}, \text { when } \mathbf{s}=(111)^{\top} .
$$

On the other hand, the $\mathbb{Z}_{4}$ symmetry is generated by the second column of $C$ since

$$
D \mathbf{s}=4\left(\begin{array}{l}
0 \\
1
\end{array}\right)=0 \bmod 4, \text { when } \mathbf{s}=(210)^{\top},
$$

which means $\frac{1}{4} \mathbf{s}$ is a nontrivial solution to (2.5) associated to the nontrivial factor 4 in $\operatorname{SNF}(D)$. For this reason, when we say $\mathbf{s}$ is the generator of $\mathbb{Z}_{d}$, we adopt the convention that the corresponding rephasing transformation (2.3) uses the vector divided by the factor $d$ as $A=\frac{1}{d}$ s.

\subsection{Charges are not unique}

The charges that generate each discrete symmetry are not unique because $R$ and $C$ in (2.8) are not unique. For example, the following $R, C$ also diagonalize $D$ :

$$
R=\left(\begin{array}{ll}
1 & 0 \\
2 & 1
\end{array}\right), C=\left(\begin{array}{ccc}
0 & 1 & 1 \\
0 & 0 & 1 \\
1 & -1 & 1
\end{array}\right)
$$

Therefore the charge $\mathbf{s}^{\prime}=(1,0,-1)^{\top}$ could be equally used instead of $(2.13)$ as a generator of $\mathbb{Z}_{4}$. As a necessary condition, a charge $\mathbf{s}$ should be a vector of relatively prime integers because $C$ is a invertible integer matrix which should have determinant \pm 1 . If a column has a common factor $k,|k| \neq 1$, then the determinant is also divisible by $k$.

The first freedom for charges is calculation modulo $k$. In our example, we often can make calculations in $\mathbb{Z}_{4}$, modulo 4 . For example,

$$
\mathbf{s}=3\left(\begin{array}{l}
2 \\
1 \\
0
\end{array}\right) \quad \bmod 4=\left(\begin{array}{c}
2 \\
-1 \\
0
\end{array}\right)
$$


can be equally used as charge instead of (2.13) because $a^{3}$ is a generator of $\mathbb{Z}_{4}$ generated by $a$ (in multiplicative notation). The vector

$$
\mathbf{s}=2\left(\begin{array}{l}
2 \\
1 \\
0
\end{array}\right) \quad \bmod 4=\left(\begin{array}{l}
0 \\
2 \\
0
\end{array}\right)
$$

can not be used as a generator because it can be divided by 2 and it only generates the $\mathbb{Z}_{2}$ subgroup of $\mathbb{Z}_{4}$, as $a^{2}$ in $\mathbb{Z}_{4}$ generated by $a$. One can check only $\mathbb{Z}_{2}$ is generated by following the action (2.3) with $A=\frac{1}{4}$ s.

In the presence of one or more $\mathrm{U}(1)$ symmetries, we can also add any combination of their charges at will. For example,

$$
\mathbf{s}=\left(\begin{array}{l}
2 \\
1 \\
0
\end{array}\right) \rightarrow \mathbf{s}^{\prime}=\left(\begin{array}{l}
2 \\
1 \\
0
\end{array}\right)-\left(\begin{array}{l}
1 \\
1 \\
1
\end{array}\right)=\left(\begin{array}{c}
1 \\
0 \\
-1
\end{array}\right),
$$

also works as a charge vector. On the other hand,

$$
\mathbf{s}^{\prime \prime}=2\left(\begin{array}{l}
2 \\
1 \\
0
\end{array}\right)-\left(\begin{array}{l}
1 \\
1 \\
1
\end{array}\right)=\left(\begin{array}{c}
3 \\
1 \\
-1
\end{array}\right),
$$

leads to

$$
D \mathbf{s}^{\prime \prime}=\left(\begin{array}{l}
0 \\
8
\end{array}\right) .
$$

This means $\mathbf{s}^{\prime \prime}$ only generates $\mathbb{Z}_{2}$ instead of $\mathbb{Z}_{4}$ and cannot be used as a charge vector, although it consists of relatively prime integers. One can see this more clearly by checking the transformation (2.3) with $A=\frac{1}{4} \mathbf{s}^{\prime \prime}$, with the result being valid modulo the $\mathrm{U}(1)$ symmetry. (Note that $\mathbb{Z}_{4}$ would be still generated if we had used $A=\frac{1}{8} \mathbf{s}^{\prime \prime}$ instead.)

\subsection{Notation}

In general, we can analyze the existence of discrete symmetries defined by $D$ in terms of the linear equation

$$
D A=B,
$$

where $A \in \mathbb{Z}^{n_{F}}$ and $B \in \mathbb{Z}^{n_{t}}$, where $n_{F}$ and $n_{t}$ are the number of (complex) fields and the number of phase sensitive terms in the Lagrangian, respectively. (Pairs of different hermitean conjugate terms are counted as one term.) Compared to ref. [15], we are already rescaling the equation (2.20) by $2 \pi$; see (2.3).

The existence of the SNF for $D$,

$$
\operatorname{SNF}(D)=R D C=\operatorname{diag}\left(d_{1}, d_{2}, \cdots, d_{n}, 0, \cdots, 0\right),
$$

where each $d_{i}$ divides $d_{i+1}$, allows us to diagonalize eq. (2.20) into $D^{\prime} A^{\prime}=B^{\prime}$, where $D^{\prime}=\operatorname{SNF}(D)$; cf. eq. (2.9). Given the SNF, we conclude that

$$
D \mathbf{s}^{(i)}=d_{i} \mathbf{w}^{(i)},
$$


where $\mathbf{s}^{(i)}, \mathbf{w}^{(i)}$ correspond to the $i$-th column of $C, R^{-1}$, respectively. They are generators of the lattices $\mathbb{Z}^{n_{F}}$ and $\mathbb{Z}^{n_{t}}$, respectively. Obviously, we get $D \mathbf{s}^{(i)}=\mathbf{0}$, for $i>\operatorname{rank} D$. The decomposition thus signals a symmetry $\mathbb{Z}_{d_{1}} \times \mathbb{Z}_{d_{1}} \times \cdots \times \mathbb{Z}_{d_{n}} \times[\mathrm{U}(1)]^{k}$, where $k$ is the number of zero factors. Each $\mathbb{Z}_{d_{i}}$ is generated by charge vector $\mathbf{s}^{(i)}$ which is related to the actual rephasing transformation (2.3) with phases (divided by $2 \pi$ )

$$
A=\frac{1}{d_{i}} \mathbf{s}^{(i)} .
$$

In general, we denote the rows of $D$ by

$$
D=\left(\begin{array}{c}
\mathbf{d}^{(1)} \\
\mathbf{d}^{(2)} \\
\vdots \\
\mathbf{d}^{\left(n_{t}\right)}
\end{array}\right)
$$

The rank of $D$ is the number of linearly independent (see section 2.4) rows (or columns) of $D$ and it can be counted as the number of nonzero factors in the SNF of $D$. Often, we will use matrices of full rank and denote its rank as $n$. For such a matrix, its SNF is

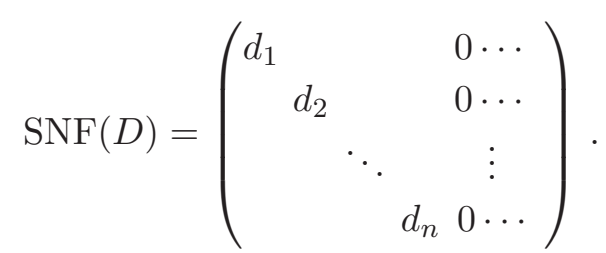

The following proposition will be useful.

Proposition. If, by adding a row $\mathbf{u}$ to a matrix $D$ of $n$ rows, its SNF remains the same, except for an additional zero row, then $\mathbf{u}$ is an integer linear combination of the rows of D. The converse also holds.

We can see the converse is trivially valid because the last row $\mathbf{u}$ can be removed by elementary row operations. The proof of the main proposition is given in appendix A.

\subsection{Linear independence}

For real or complex vectors spaces (a vector space over any field) such as $\mathbb{R}^{n}$ or $\mathbb{C}^{n}$ we know that the following statements are equivalent: (i) a set $\left\{\mathbf{u}_{1}, \mathbf{u}_{2}, \cdots, \mathbf{u}_{k}\right\}$ is linearly dependent; (ii) one of the vectors in the set $\left\{\mathbf{u}_{1}, \mathbf{u}_{2}, \cdots, \mathbf{u}_{k}\right\}$ can be written as a linear combination of the rest of vectors.

Here we need to consider vectors of integer components and they live in $\mathbb{Z}^{n}$. These spaces are similar to vector spaces (they are called modules) and most of the usual properties of vectors spaces remain. The equivalence of properties (i) and (ii), however, does not hold. In this article, when we refer to linear independence, we will refer to such a property over the integers (in $\mathbb{Z}^{n}$ ) if not specified otherwise.

As an example, let us take three vectors in $\mathbb{Z}^{3}$ :

$$
\mathbf{u}_{1}=(2,-1,-1), \mathbf{u}_{2}=(-1,2,-1), \mathbf{u}_{3}=(2,-2,0) .
$$


These three vectors are linearly dependent since

$$
2 \mathbf{u}_{1}+(-2) \mathbf{u}_{2}+(-3) \mathbf{u}_{3}=\mathbf{0} .
$$

However, we cannot write any $\mathbf{u}_{i}$ as an integer linear combination of two other vectors.

The SNF can be used to extract these properties as follows:

$$
\begin{aligned}
\operatorname{SNF}\left(\mathbf{u}_{1}, \mathbf{u}_{2}, \mathbf{u}_{3}\right) & =\operatorname{diag}(1,1,0) \\
\operatorname{SNF}\left(\mathbf{u}_{1}, \mathbf{u}_{2}\right) & =\operatorname{diag}(1,3), \\
\operatorname{SNF}\left(\mathbf{u}_{1}, \mathbf{u}_{3}\right) & =\operatorname{diag}(1,2)
\end{aligned}
$$

where $\operatorname{SNF}\left(\mathbf{u}_{1}, \mathbf{u}_{2}\right)$ denote the Smith normal form of a matrix composed of rows $\mathbf{u}_{1}, \mathbf{u}_{2}$. The linear dependence of three $\mathbf{u}_{i}$ can be seen in the zero factor of the first equation: the rank is two. The sets $\left\{\mathbf{u}_{1}, \mathbf{u}_{2}\right\}$ and $\left\{\mathbf{u}_{1}, \mathbf{u}_{3}\right\}$ are linearly independent but they generate different subspaces (lattices) of $\mathbb{Z}^{3}$ and their SNF are different.

\subsection{Height of vector}

Take a vector (row) $\mathbf{u}$ of a $D$-matrix derived from phase sensitive terms in a Lagrangian. We use the quantity $\left(l_{1}\right.$-norm)

$$
h(\mathbf{u})=\sum_{i}\left|u_{i}\right|
$$

to classify vectors into different heights. We will call $\mathbf{u}$ as a height- $k$ vector when $h(\mathbf{u})=k$. This quantity counts the number of phase sensitive fields in the Lagrangian term associated to $\mathbf{u}$. For example, for a potential $V$ composed of scalar fields $\phi_{i}$, we can associate

$$
\begin{aligned}
& \phi_{1}^{\dagger} \phi_{2} \subset \mathscr{L} \Longrightarrow \mathbf{u}=(-1,1,0, \cdots), \\
& \phi_{1}^{\dagger} \phi_{2} \phi_{1}^{\dagger} \phi_{3} \subset \mathscr{L} \Longrightarrow \mathbf{u}=(-2,1,1,0, \cdots), \\
& \phi_{1}^{\dagger} \phi_{2} \phi_{3}^{\dagger} \phi_{1} \subset \mathscr{L} \Longrightarrow \mathbf{u}=(0,1,-1,0, \cdots) .
\end{aligned}
$$

In the first two examples, $h(\mathbf{u})$ corresponds to the number of fields whereas in the third example the term is not sensitive to the phase of $\phi_{1}$. Elementary row operations does not maintain the height fixed but this classification will still be useful; see e.g. section 5.2.

\section{Matching up symmetries}

From this point on, we will concentrate on the N-Higgs-doublet models with quarks, which can be divided into two sectors: the Yukawa interactions and the Higgs potential. We denote the $D$-matrix for the terms in the Yukawa interactions and in the potential as $D_{\text {full }}^{Y}$ and $D^{V}$, respectively. Applications to the lepton sector or to other models can be considered in an analogous manner. We only consider renormalizable terms and assume that discrete symmetries are valid up to very high energies (e.g., remnants of local gauge symmetries) so that no appreciable breaking is expected through nonrenormalizable terms. We consider the most general Yukawa interactions in the form [15]

$$
-\mathcal{L}_{Y}=\Gamma_{j_{L} j_{d}}^{\left(j_{\phi}\right)} \bar{Q}_{L j_{L}} \phi_{j_{\phi}} d_{R j_{d}}+\Delta_{j_{L} j_{u}}^{\left(j_{\phi}\right)} \bar{Q}_{L j_{L}} \tilde{\phi}_{j_{\phi}} u_{R j_{u}}+\text { h.c. }
$$

where $j_{L}, j_{d}, j_{u}$ run from 1 to 3 and $j_{\phi}$ runs from 1 to $N$. 
The current knowledge is summarized for small $N$ in the following table containing the bounds for the order of realizable abelian symmetries in the potential $\left(G_{\phi}\right)$ and in the Yukawa interactions $\left(G_{q}\right)$ :

\begin{tabular}{|r|ccccc|}
\hline$N$ & 2 & 3 & 4 & 5 & 6 \\
\hline$\left|G_{q}\right| \leq$ & 3 & 5 & 8 & 12 & $\underline{16}$ \\
$\left|G_{\phi}\right| \leq$ & 2 & 4 & 8 & 16 & 32 \\
\hline
\end{tabular}

The upper bounds in the last row where shown in [39] and they correspond to the exact upper boundary. The upper bounds in the first row were proven in [15] and all cases up to $N=5$ were explicitly shown to be realizable (we mark the unproven case underlined). Algebraically, we have [15, 39]

$$
\begin{aligned}
\left|G_{q}\right|_{\text {bound }} & = \begin{cases}n^{2} / 3, & \text { if } n \text { is divisible by } 3, \\
\left(n^{2}-1\right) / 3, & \text { if } n \text { is not divisible by } 3\end{cases} \\
\left|G_{\phi}\right|_{\max } & =2^{N-1},
\end{aligned}
$$

where $n=N+1$. As before, $\left|G_{\phi}\right|_{\max }$ is the exact boundary while $\left|G_{q}\right|_{\text {bound }}$ is an upper bound. We can see that the maximal group size grows much quickly for the potential than for the Yukawa interactions. Qualitatively, that happens because as $N$ grows the number of Lagrangian terms in the potential grows much quickly $\left(\sim N^{4}\right)$ than the number of Yukawa terms with three families of quarks $(\sim N)$.

We now seek conditions for a given abelian symmetry in one sector to be compatible with the symmetry in the other sector. Let us denote the symmetries in the Yukawa sector and in the potential as $G_{q}$ and $G_{\phi}$, respectively. Often these groups will refer to abstract groups and we disregard the automatic symmetries $\mathrm{U}(1)_{B}, \mathrm{U}(1)_{Y}$, and others that might appear. For example, if a field does not appear in a sector of the Lagrangian, then its rephasing is automatically a symmetry. The true symmetry of the theory is $G_{F}=\tilde{G}_{q} \cap \tilde{G}_{\phi} \cdot{ }^{1}$ Moreover, $G_{q} \neq G_{F}$ or $G_{\phi} \neq G_{F}$ (not isomorphic) is naturally possible only if they arise accidentally from $G_{F}$ or a common smaller symmetry contained in $G_{F}$. We also note that in this specific setting (NHDMs), $G_{F}$ needs to be entirely broken after EWSB to allow nonzero and nondegenerate quark masses with mixing [41, 42].

To set the notation for applying the Smith normal form method, we use the basis with ordering $\left(\phi_{a} ; Q_{i L} ; d_{i R} ; u_{i R}\right)$, corresponding to $n_{F}=N+9$ complex fields (columns of $\left.D_{\text {full }}^{Y}, D^{V}\right)$. However, the relevant information from $D_{\text {full }}^{Y}$ on nontrivial symmetries may be extracted from a reduced matrix $D^{Y}$, which depends only on the $N+3$ fields $\left(\phi_{a} ; Q_{i L}\right)$. A different notation was used in ref. [15] where the reduced matrix was denoted by $\tilde{D}$ whereas the full matrix was simply $D$. We also note that, for $N \geq 6$, the rows of $D^{Y}$ can not be generic but we can exclude cases by assuming generic matrices. Now, we proceed to analyze the compatibility of $D^{Y}$ and $D^{V}$.

\footnotetext{
${ }^{1}$ For this identification, we need to consider $\tilde{G}_{q}$ and $\tilde{G}_{\phi}$ as full subgroups inside $[\mathrm{U}(1)]^{n_{F}}$, the rephasing symmetries of all $n_{F}$ complex fields involved. Therefore, $\tilde{G}_{q}=G_{q}$ but $\tilde{G}_{\phi}$ contains the additional rephasing symmetries for the fermion fields besides $G_{\phi}$. The distinction may be important in some situations; see remarks in appendix B.
} 
The minimal number of rows (phase sensitive terms in the Lagrangian) necessary to sustain discrete symmetries in $D^{Y}$ and $D^{V}$ are $n=N+1$ and $n-2$, respectively. To analyze the symmetry of the whole theory we also define the $D$-matrix for the whole theory as

$$
D^{T}=\left(\frac{D^{Y}}{D^{V}}\right) .
$$

If $D^{Y}$ and $D^{V}$ have $n$ and $n-2$ rows, respectively, then $D^{T}$ has $2 n-2$ rows.

For the minimal number of rows, the generic structure of $D^{Y}$ and $D^{V}$ reads

$$
D^{Y}=\left(\begin{array}{c|c}
\mathbf{p}_{1} & \mathbf{q}_{1} \\
\mathbf{p}_{2} & \mathbf{q}_{2} \\
\vdots & \vdots \\
\mathbf{p}_{n} & \mathbf{q}_{n}
\end{array}\right), \quad D^{V}=\left(\begin{array}{c|c}
\mathbf{r}_{1} & \mathbf{0}_{3} \\
\mathbf{r}_{2} & \mathbf{0}_{3} \\
\vdots & \vdots \\
\mathbf{r}_{n-2} & \mathbf{0}_{3}
\end{array}\right)
$$

The p-, q- and $\mathbf{r}$-vectors have length $n-1(N), 3$ and $n-1$, respectively. The p-vectors are of the form $(1,-1,0, \ldots)$, or permutations, the $\mathbf{q}$-vectors are of the form $(1,-1,0)$, or any permutation, and the $\mathbf{r}$-vectors are made up of one $\mathbf{p}$-vector or the sum of two $\mathbf{p}$-vectors. The columns of zeros denoted by $\mathbf{0}_{3}=(0,0,0)$ correspond to three fields $Q_{i L}$ which do not appear in the potential. If we use the classification of section 2.5 , $\mathbf{p}$ - and $\mathbf{q}$-vectors are of height 2 (one of them can be of height 0 ) and $\mathbf{r}$-vector are of heights 2 or 4 . Given the automatic conservation of $\mathrm{U}(1)_{Y}$ and $\mathrm{U}(1)_{B}$, when we sum p- or q-vectors, their height always change in units of two. For example, by summing two q-vectors, we can obtain vectors of heights 0,2 or 4 .

We start by analyzing the conditions for faithful compatibility, i.e., $G_{q} \simeq G_{\phi}$ (isomorphic). We say $G_{q}$ (Yukawa) and $G_{\phi}$ (potential) are faithfully compatible symmetries in the whole theory if all the integers that appear in the $\mathrm{SNF}$ of $D^{Y}, D^{V}$ and $D^{T}$ are the same:

$$
\begin{aligned}
& \text { (I.Y) } \operatorname{SNF}\left(D^{Y}\right)=\left(\begin{array}{llllll}
d_{1} & & & & 0 & 0 \\
& d_{2} & & & 0 & 0 \\
& & \ddots & & \vdots & \vdots \\
& & & d_{n} & 0 & 0
\end{array}\right) \text {, }
\end{aligned}
$$

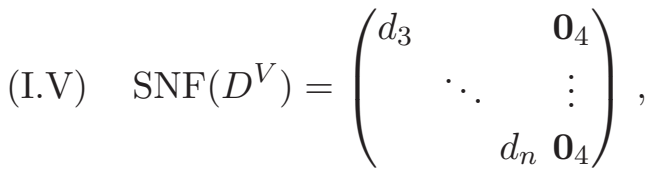

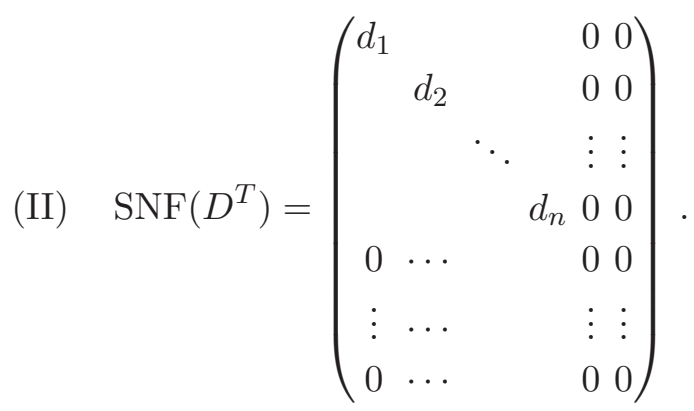


Often most of the factors $d_{i}$ will be unity. In particular, we must necessarily have $d_{1}=d_{2}=$ 1. Generalization to theories with more sectors can be done by considering the separation of the theory into one sector and the rest.

If the factors appearing in (I.Y), (I.V) and (II) do not all match but (II) still contains non-unit integers, we still say $G_{q}$ and $G_{\phi}$ are compatible and the symmetry of the whole theory $\left(G_{F}\right)$ is different from the symmetry of one or both of its subsectors $\left(G_{q}\right.$ or $\left.G_{\phi}\right)$. In any of these cases, $G_{F}$ is the true symmetry of the theory if there are no additional $G_{F}$-symmetric terms that reduces $G_{q}$ or $G_{\phi}$ (and possibly $G_{F}$ ). If $G_{q}$ or $G_{\phi}$ changes after the addition of $G_{F}$-symmetric terms, we say they are not compatible. In practice, we can begin with some $G_{\phi}$ or $G_{q}$ and start to add $G_{F}$-symmetric terms if possible. If the process stops with some finite $G_{F}$, then the groups $G_{q}$ and $G_{\phi}$ in the last step are compatible. Of course, the process may continue until we have no symmetry. Natural compatibility that is not faithful may happen in two situations: either the symmetry in some subsector contains accidental symmetries not present in $G_{F}$ or only a subgroup of $G_{F}$ is faithfully represented in some subsector. One example of the former case can be seen in section 6.3 where $G_{F} \simeq G_{\phi}=\mathbb{Z}_{8}$ but $G_{q}=\mathrm{U}(1) \supset G_{F}$. Appendix B contains an example for the second case. In general settings, not faithful natural compatibility is difficult to characterize and thus we will concentrate on the faithfully compatible cases.

Let us return to the conditions in (3.6) and analyze its consequences. Such conditions imply that the rank of $D^{T}$ are the same as the rank of $D^{Y}$ and we can conclude that the $\mathbf{r}$ vectors in $D^{V}$ should be particular linear combinations of the $\mathbf{p}$-vectors of $D^{Y}$, constrained by the condition that the contribution from the $\mathbf{q}$-vectors should vanish. Let us assume, without loss of generality, that $\left\{\mathbf{q}_{1}, \mathbf{q}_{2}\right\}$ are linearly independent. By row operations we can transform $D^{Y}$ in (3.5) to

$$
D^{Y} \sim\left(\begin{array}{c|c}
\mathbf{p}_{1} & \mathbf{q}_{1} \\
\mathbf{p}_{2} & \mathbf{q}_{2} \\
\mathbf{p}_{3}^{\prime} & \mathbf{0}_{3} \\
\vdots & \vdots \\
\mathbf{p}_{n}^{\prime} & \mathbf{0}_{3}
\end{array}\right) .
$$

From the structure of $\mathbf{q}$-vectors, we conclude that the $\mathbf{p}_{i}^{\prime}$ vectors are made of at most three $\mathbf{p}$-vectors and it should be of height at most 6 . Full compatibility and the proposition of section 2.3 then requires that $\mathbf{p}_{i}^{\prime}$ be integer linear combinations of $\mathbf{r}_{i}$ and the reverse should be also true. Therefore we conclude that the following matrices should be equivalent by row operations for faithful compatibility:

$$
\left(\begin{array}{c}
\mathbf{p}_{3}^{\prime} \\
\vdots \\
\mathbf{p}_{n}^{\prime}
\end{array}\right) \sim\left(\begin{array}{c}
\mathbf{r}_{1} \\
\vdots \\
\mathbf{r}_{n-2}
\end{array}\right)
$$

\section{Maximal symmetries for three Higgs doublets}

Before considering the three-Higgs-doublet case, let us review the simpler $2 \mathrm{HDM}$ with $\mathbb{Z}_{2}$ symmetry. We know that the simple $\mathbb{Z}_{2}$ symmetry can be implemented in the $2 \mathrm{HDM}$ to 
naturally suppress flavor changing neutral currents [22], and it is often used as a benchmark model for testing 2HDMs [43]. That implementation, actually, leads to an accidental PecceiQuinn symmetry in the Yukawa sector which is only broken to $\mathbb{Z}_{2}$ in the Higgs potential through the term $\left(\phi_{1}^{\dagger} \phi_{2}\right)^{2}$. (See end of sectionIV.B in ref. [30].) A true 2HDM with $\mathbb{Z}_{2}$ symmetry $^{2}$ in both Yukawa sector and Higgs potential can be constructed [30] but it requires flavor dependent $\mathbb{Z}_{2}$ charges and thus to potentially harmful FCNC interactions unless the non-SM scalar bosons are very heavy.

Returning to three-Higgs-doublets, $N=3$, we can see in table (3.2) that $\left|G_{q}\right|_{\max }=$ $5 \geq\left|G_{\phi}\right|_{\max }=4$. Thus the abelian symmetries $G_{F}$ which are realizable for 3HDM should have order equal or less than 4 . We show here that $\left|G_{F}\right|=4$ is realizable and we explicitly give the backbone structure from which the whole theory can be reconstructed [15]. Let us recall that the $D$-matrix for Yukawa interactions in this case has the form (3.5),

$$
D^{Y}=\left(\begin{array}{c|c}
\mathbf{p}_{1} & \mathbf{q}_{1} \\
\mathbf{p}_{2} & \mathbf{q}_{2} \\
\mathbf{p}_{3} & \mathbf{q}_{3} \\
\mathbf{p}_{4} & \mathbf{q}_{4}
\end{array}\right) .
$$

The $\mathbf{p}$-vectors (q-vectors) correspond to the fields $\phi\left(Q_{L}\right)$ and have the form $(1,-1,0)$ or similar with permuted entries. There are 6 possibilities with only three nonparallel vectors, which we denote as being of distinct type:

$$
\mathbf{q}_{i} \in\{ \pm(1,-1,0), \pm(0,1,-1), \pm(1,0,-1)\}
$$

Only two of them are linearly independent. We have seen in [15] that $\left|G_{q}\right|_{\max }=5$ is achieved only when the q-vectors are picked most evenly among the different types, i.e., the number $n_{i}$ of a type $i$ vector should be $\left(n_{1} n_{2} n_{3}\right)=(211)$ and the order of the group is bounded by $m=n_{1} n_{2}+n_{2} n_{3}+n_{3} n_{1}=5$. To get $\left|G_{q}\right|=4$, we can allow $\left(n_{1} n_{2} n_{3}\right)=(211)$ or $\left(n_{1} n_{2} n_{3}\right)=(220)$. Since $\mathbf{p}$-vectors and $\mathbf{q}$-vectors are the same in this case, the same distribution of different types also applies to the $\mathbf{p}$-vectors.

By row operations, we can obtain the form (3.7) which, in this case, reads

$$
D^{Y} \sim\left(\begin{array}{c|c}
\mathbf{p}_{1} & \mathbf{q}_{1} \\
\mathbf{p}_{2} & \mathbf{q}_{2} \\
\mathbf{p}_{3}^{\prime} & \mathbf{0} \\
\mathbf{p}_{4}^{\prime} & \mathbf{0}
\end{array}\right)
$$

where $\mathbf{p}_{i}^{\prime}$ are at most height- 6 vectors, as they are integer combinations of $\mathbf{p}_{i}$ and $\mathbf{p}_{1}, \mathbf{p}_{2}$ (we are assuming $\mathbf{q}_{1}, \mathbf{q}_{2}$ nonparallel). When calculating the SNF form of $D^{Y}$, we can see the nontrivial integers come from the submatrix $\left(\mathbf{p}_{3}^{\prime}, \mathbf{p}_{4}^{\prime}\right)$. Analogously, the reduced $D$-matrix of the potential is

$$
D^{V}=\left(\begin{array}{l|l}
\mathbf{r}_{1} & \mathbf{0} \\
\mathbf{r}_{2} & \mathbf{0}
\end{array}\right) .
$$

\footnotetext{
${ }^{2} \mathrm{An}$ accidental CP symmetry is always present in the potential [24-29].
} 
We know that the vectors $\mathbf{r}_{1}, \mathbf{r}_{2}$ are at most height- 4 vectors. The compatibility condition in (3.8) requires that $\mathbf{p}_{3}^{\prime}, \mathbf{p}_{4}^{\prime}$ be integer linear combinations of $\mathbf{r}_{1}, \mathbf{r}_{2}$ and vice-versa. Let us see if that is possible for $\left|G_{F}\right|=4$.

To satisfy the conditions (I) of (3.6), the SNFs of the sectors $\left(\mathbf{r}_{1}, \mathbf{r}_{2}\right),\left(\mathbf{p}_{3}^{\prime}, \mathbf{p}_{4}^{\prime}\right)$ should contain the same non-unit integers that multiply to 4 . The only possibilities are one unit integer 4 or two non-unit integers $(2,2)$. These possibilities correspond to the symmetries $\mathbb{Z}_{4}$ and $\mathbb{Z}_{2} \times \mathbb{Z}_{2}$, respectively. In the potential, these possibilities correspond to the backbone structures [15]

$$
\begin{aligned}
\mathbb{Z}_{4}: & D^{V}=\left(\begin{array}{ccc|c}
2 & -1 & -1 & \mathbf{0} \\
0 & 2 & -2 & \mathbf{0}
\end{array}\right), \\
\mathbb{Z}_{2} \times \mathbb{Z}_{2}: & D^{V}=\left(\begin{array}{ccc|c}
2 & -2 & 0 & \mathbf{0} \\
0 & 2 & -2 & \mathbf{0}
\end{array}\right) .
\end{aligned}
$$

Other possibilities amount to relabeling of Higgs doublets. These structures lead to the charges

$$
\begin{aligned}
\mathbb{Z}_{4}: & \mathbf{s}^{V}=(1,2,0 ; * * *)^{\top} \sim(0,1,-1 ; * * *)^{\top}, \\
\mathbb{Z}_{2} \times \mathbb{Z}_{2}: & \mathbf{s}^{V}=(1,0,0 ; * * *)^{\top} \otimes(0,0,1 ; * * *)^{\top},
\end{aligned}
$$

where we marked the undefined $Q_{i L}$ charges by $*$ and subtracted one unit of the conserved charge $(111 ; \mathbf{0})$ on $\mathbf{s}^{V}$ of $\mathbb{Z}_{4}$. Notice that we use $\mathbf{s}$ instead of $\tilde{\mathbf{s}}$ when compared to ref. [15].

Let us now analyze if $\mathbf{p}_{3}^{\prime}, \mathbf{p}_{4}^{\prime}$ can be of height- 6 . All height- 6 vectors in this case are $(3,-2,-1),(3,-3,0)$ or vectors with permuted entries. By applying a height-6 vector (whose sum of components are zero) to the $\mathbb{Z}_{4}$-charge in (4.6), it is not possible to obtain zero or a multiple of 4 . Analogously, for $\mathbb{Z}_{2} \times \mathbb{Z}_{2}$-charge it is not possible to obtain a pair $\left(a_{1}, a_{2}\right)$ where each of $a_{i}$ is zero or multiple of 2 . Therefore $\mathbf{p}_{3}^{\prime}, \mathbf{p}_{4}^{\prime}$ should be of height-4 or less. But from condition (I.Y) of (3.6) neither of them can be of height-2 (it leads to unit factors in SNF). Thus we conclude they must be both of height-4. Therefore, $\left(\mathbf{p}_{3}^{\prime}, \mathbf{p}_{4}^{\prime}\right)$ should be also of the form (4.5).

Now, we can check that the only height- 4 vectors that give zero or multiples of 4 after applying the $\mathbb{Z}_{4}$-charge are the two rows of $D^{V}\left(\mathbb{Z}_{4}\right)$ in (4.5). For $\mathbb{Z}_{2} \times \mathbb{Z}_{2}, \mathbf{p}_{3}^{\prime}$, $\mathbf{p}_{4}^{\prime}$ should correspond to integer combinations of $\mathbf{r}_{i}$. Then $\mathbf{p}_{3}^{\prime}, \mathbf{p}_{4}^{\prime}$ are two nonparallel vectors of the form $(2,-2,0)$ or permuted entries. In either case, given that the SNF form of $\left(\mathbf{p}_{3}^{\prime}, \mathbf{p}_{4}^{\prime}\right)$ should also correspond to a finite symmetry, we conclude that $\mathbf{p}_{3}^{\prime}, \mathbf{p}_{4}^{\prime}$ must correspond to the rows of $D^{V}$ in (4.5), except for a possible interchange or overall minus sign.

The following step would be to list all $D^{Y}$ matrices (4.1) that are compatible with (4.3), where $\left(\mathbf{p}_{3}^{\prime}, \mathbf{p}_{4}^{\prime}\right)$ are of the form (4.5). Since this backward analysis would be lengthy, we employ a different strategy in appendix $\mathrm{C}$ to characterize all possible matrices $D^{Y}$ of the form (4.1) which are compatible with $D^{V}$ in (4.5). We find only one backbone structure for both symmetries $\mathbb{Z}_{4}$ and $\mathbb{Z}_{2} \times \mathbb{Z}_{2}$. We list them in the following together with their complete charges. By using charges, Yukawa textures can be recovered from the recipe given in section 6.2 of ref. [15]. After applying the recipe, we need to check if the theory realizes the discrete symmetry or if it is invariant by a larger $\mathrm{U}(1)$ symmetry containing the discrete symmetry as a subgroup. The former is true if $D^{Y} \mathbf{s} \neq \mathbf{0}$. 


\section{$4.1 \mathbb{Z}_{4}-3 \mathrm{HDM}$}

The unique backbone structure for $\mathbb{Z}_{4}-3 \mathrm{HDM}$ is

$$
D^{Y}=\left(\begin{array}{ccc|ccc}
1 & -1 & 0 & 1 & -1 & 0 \\
1 & -1 & 0 & -1 & 1 & 0 \\
0 & 1 & -1 & 0 & 1 & -1 \\
1 & 0 & -1 & 0 & -1 & 1 \\
0 & 1 & -1 & -1 & 0 & 1 \\
1 & 0 & -1 & 1 & 0 & -1
\end{array}\right), \quad D^{V}=\left(\begin{array}{ccc}
2 & -2 & 0 \\
1 & 1 & -2
\end{array}\right)
$$

where we reordered the matrix in (4.5) for compatibility and suppressed the automatic zeros in $D^{V}$ for simplicity. The details can be found in appendix C. We should note that $D^{Y}$ can have 4 rows to sustain a finite symmetry but we discover that the possible backbone structures for $D^{Y}$ which contains 4 rows are all compatible with the structure above. Different possibilities only amounts to reordering $\phi_{a}$ and $Q_{i L}$ fields. One can also check that if we write $D^{Y}$ in the form (3.7), we obtain exactly $D^{V}$ in the lower rows.

The $\mathbb{Z}_{4}$-charges arising from (4.7) reads

$$
\mathbf{s}_{\mathbb{Z}_{4}}=(1,-1,0 ;-1,1,0) .
$$

We can see these charges in the $\phi$-sector are analogous to (4.6) after reordering.

\section{$4.2 \quad \mathbb{Z}_{2} \times \mathbb{Z}_{2}-3$ HDM}

There is only one backbone structure for $\mathbb{Z}_{2} \times \mathbb{Z}_{2}-3 \mathrm{HDM}$ :

$$
D^{Y}=\left(\begin{array}{ccc|ccc}
1 & -1 & 0 & 1 & -1 & 0 \\
1 & -1 & 0 & -1 & 1 & 0 \\
0 & 1 & -1 & 0 & 1 & -1 \\
0 & 1 & -1 & 0 & -1 & 1 \\
1 & 0 & -1 & -1 & 0 & 1 \\
1 & 0 & -1 & 1 & 0 & -1
\end{array}\right), \quad D^{V}=\left(\begin{array}{ccc}
2 & -2 & 0 \\
0 & 2 & -2 \\
2 & 0 & -2
\end{array}\right)
$$

The corresponding charges are

$$
\mathbf{s}_{\mathbb{Z}_{2} \times \mathbb{Z}_{2}}=(1,0,0 ; 1,0,0) \otimes(0,0,1 ; 0,0,1) .
$$

One can also check that if we write $D^{Y}$ in the form (3.7), we obtain exactly $D^{V}$ in the lower rows.

\section{$5 \quad$ Discarding maximal symmetries}

We show here that the abelian symmetry in the table of (3.2), with smallest order for each $N$, is not compatible in both the Yukawa sector and the Higgs potential for $N \geq 4$, and its imposition leads to a continuous symmetry in the potential.

We see in the table of (3.2) that $\left|G_{\phi}\right|_{\max } \geq\left|G_{q}\right|_{\text {bound }}$ for $N \geq 4$. The maximal order of the groups allows, in principle, a common abelian group $G_{F}=G_{\phi}=G_{q}$ with order $\left|G_{F}\right|=\left|G_{q}\right|_{\max } \leq\left|G_{q}\right|_{\text {bound. }}$. We will show that $G_{\phi}$ compatible with $\left.G_{q}\right|_{\max }$ can not be found. 


\subsection{Discarding maximal symmetries for $N=4$ and $N=5$}

Let us take $N=4$ and $N=5$ explicitly. We begin with $N=4$ where we investigate if $\mathbb{Z}_{8}$ symmetry is realizable in the whole $4 \mathrm{HDM}$ theory. From the backbone structure of the Yukawa interactions of $\mathbb{Z}_{8}-4 \mathrm{HDM}$ in ref. [15],

$$
D_{N=4}^{Y}\left(\mathbb{Z}_{8}\right)=\left(\begin{array}{cccc|ccc}
0 & 1 & -1 & 0 & 1 & 0 & -1 \\
0 & 1 & 0 & -1 & -1 & 1 & 0 \\
1 & -1 & 0 & 0 & 0 & 1 & -1 \\
-1 & 0 & 1 & 0 & 1 & 0 & -1 \\
-1 & 0 & 0 & 1 & -1 & 1 & 0
\end{array}\right)
$$

we can find the following $\mathbb{Z}_{8}$ charges for $\left(\phi ; Q_{L}\right)$ fields:

$$
\mathbf{s}_{\mathbb{Z}_{8}}=(-1,1,4,0 ; 1,0,-2)^{\top},
$$

Note that we use a different convention from [15] where we can find (without the prime)

$$
\mathbf{s}_{\mathbb{Z}_{8}}^{\prime}=(0,2,1,-3 ;-1,2,0)^{\top} .
$$

We note that

$$
\mathbf{s}_{\mathbb{Z}_{8}}-\mathbf{s}_{\mathbb{Z}_{8}}^{\prime}=(-1,-1,3,3 ; 2,-2,-2)^{\top} \sim(0,0,4,4 ; 4,0,0)^{\top},
$$

where in the last equivalence we have used invariance by $\mathrm{U}(1)_{Y}$ and $\mathrm{U}(1)_{B}$. One can check that

$$
D_{N=4}^{Y}\left(\mathbb{Z}_{8}\right)\left(\mathbf{s}_{\mathbb{Z}_{8}}-\mathbf{s}_{\mathbb{Z}_{8}}^{\prime}\right)=(0,-8,0,8,0)^{\top}
$$

i.e., the zero vector if the components are taken modulo 8 .

Now, analyzing the $\phi$ charges of (5.2), we can immediately see that there is no quadratic invariants of the form $\phi_{i}^{\dagger} \phi_{j}$ and the only quartic invariant terms are

$$
\left(\phi_{4}^{\dagger} \phi_{3}\right)^{2}, \phi_{1}^{\dagger} \phi_{4} \phi_{2}^{\dagger} \phi_{4}, \phi_{1}^{\dagger} \phi_{3} \phi_{2}^{\dagger} \phi_{3} \text {. }
$$

These terms lead to the $D$-matrix:

$$
D^{V}=\left(\begin{array}{cccc}
-1 & -1 & 2 & 0 \\
-1 & -1 & 0 & 2 \\
0 & 0 & 2 & -2
\end{array}\right)
$$

However, we can immediately see that the third row is the first row subtracted from the second and thus $D^{V}$ has rank two. We need at least another linearly independent row to obtain a finite symmetry. Thus the Higgs potential has an U(1) symmetry.

Let us now turn to $N=5$. The backbone structure of the Yukawa interactions for $\mathbb{Z}_{2} \times \mathbb{Z}_{6}-5 \mathrm{HDM}$ is $[15]$

$$
D_{N=5}^{Y}\left(\mathbb{Z}_{2} \times \mathbb{Z}_{6}\right)=\left(\begin{array}{ccccc|ccc}
1 & -1 & 0 & 0 & 0 & 1 & 0 & -1 \\
1 & 0 & -1 & 0 & 0 & -1 & 1 & 0 \\
0 & 0 & 0 & 1 & -1 & 0 & 1 & -1 \\
0 & 1 & 0 & -1 & 0 & 1 & 0 & -1 \\
0 & 0 & 1 & -1 & 0 & -1 & 1 & 0 \\
-1 & 0 & 0 & 0 & 1 & 0 & 1 & -1
\end{array}\right)
$$


The $\mathbb{Z}_{2} \times \mathbb{Z}_{6}$ charges can be found to be

$$
\mathbf{s}_{\mathbb{Z}_{2} \times \mathbb{Z}_{6}}=(0,1,1,0,0 ; 1,0,0)^{\top} \otimes(1,3,0,-1,0 ; 2,1,0)^{\top},
$$

where the charges refer to $\mathbb{Z}_{2}$ and $\mathbb{Z}_{6}$ respectively. We can check that the only quadratic term $\phi_{i}^{\dagger} \phi_{j}$ invariant by $\mathbb{Z}_{6}$ is $\phi_{3}^{\dagger} \phi_{5}$, but this term is not invariant by $\mathbb{Z}_{2}$. Among the quartic terms, we can find the $\mathbb{Z}_{6}$ invariants

$$
\begin{aligned}
& \phi_{i}^{\dagger} \phi_{j} \phi_{k}^{\dagger} \phi_{l}, \quad i, j, k, l=3,5, \\
& \phi_{2}^{\dagger} \phi_{i} \phi_{2}^{\dagger} \phi_{j}, \quad i, j=3,5, \\
& \phi_{1}^{\dagger} \phi_{i} \phi_{4}^{\dagger} \phi_{j}, \quad i, j=3,5, \\
& \phi_{1}^{\dagger} \phi_{4} \phi_{2}^{\dagger} \phi_{4} .
\end{aligned}
$$

Among them, the $\mathbb{Z}_{2}$ invariants are

$$
\begin{aligned}
& (i j k l)=(3535), \\
& (i j k l)=(2323),(2525), \\
& (i j k l)=(1343),(1545),
\end{aligned}
$$

where we use the shorthand $\phi_{i}^{\dagger} \phi_{j} \phi_{k}^{\dagger} \phi_{l} \sim(i j k l)$. The $D$-matrix for these terms is

$$
D^{V}=\left(\begin{array}{ccccc}
0 & 0 & -2 & 0 & 2 \\
0 & -2 & 2 & 0 & 0 \\
0 & -2 & 0 & 0 & 2 \\
1 & 0 & -2 & 1 & 0 \\
1 & 0 & 0 & 1 & -2
\end{array}\right)
$$

We can see by inspection that two rows can be eliminated by row reduction leading to a rank-3 matrix and hence the potential is $\mathrm{U}(1)$ symmetric.

It is surprising that $\mathbb{Z}_{8}$ and $\mathbb{Z}_{2} \times \mathbb{Z}_{6}$ are proven to be realizable symmetries in the potential of $4 \mathrm{HDM}$ and $5 \mathrm{HDM}$, respectively [39]. However, we have seen here that the maximal symmetries $\mathbb{Z}_{8}$ and $\mathbb{Z}_{2} \times \mathbb{Z}_{6}$ that are realizable in the Yukawa sector of $4 \mathrm{HDM}$ and 5HDM, respectively, can not be extended to the respective potentials: they lead to a continuous symmetry and to Goldstone bosons in the Higgs spectrum at the classical level. The converse also occurs: the realization of $\mathbb{Z}_{8}$ symmetry in the $4 \mathrm{HDM}$ potential also leads to a continuous symmetry in the Yukawa sector of 4HDM; see example in section 6.3. Analogous considerations apply to 5HDM.

We should also remark that when $G_{q}$ is discrete but $G_{\phi}$ is accidentally continuous, quantum corrections will generally turn the additional Goldstone bosons into pseudoGoldstone bosons. We will not focus on this possibility here.

\subsection{Discarding maximal symmetries for $N \geq 6$}

Here, we extend our results of the previous section and discard all $G_{F}=\left.G_{q}\right|_{\text {bound }}$ in table (3.2) as realizable symmetries for NHDMs for $N \geq 6$. The order for maximal $G_{q}$ 
is given in eq. (3.3). There might be symmetries in the potential $G_{\phi}$ of the same order $\left|G_{\phi}\right|=\left|G_{q}\right|_{\text {bound }}$, for $N \geq 4$ [39], but we show here that they can not be compatible.

We start by reconsidering the cases $N=4$ and $N=5$ from a different point of view. We then generalize this method to the cases $N \geq 6$. Let us transform the backbone structures for the Yukawa interactions of $\mathbb{Z}_{8}-4 \mathrm{HDM}$ and $\mathbb{Z}_{2} \times \mathbb{Z}_{6}-5 \mathrm{HDM}$ to the form (3.7):

$$
\begin{aligned}
& D_{N=4}\left(\mathbb{Z}_{8}\right) \sim\left(\begin{array}{cccc|ccc}
0 & 1 & -1 & 0 & 1 & 0 & -1 \\
0 & 1 & 0 & -1 & -1 & 1 & 0 \\
1 & -3 & 1 & 1 & 0 & 0 & 0 \\
-1 & -1 & 2 & 0 & 0 & 0 & 0 \\
-1 & -1 & 0 & 2 & 0 & 0 & 0
\end{array}\right), \\
& D_{N=5}\left(\mathbb{Z}_{2} \times \mathbb{Z}_{6}\right) \sim\left(\begin{array}{ccccc|ccc}
1 & -1 & 0 & 0 & 0 & 1 & 0 & -1 \\
1 & 0 & -1 & 0 & 0 & -1 & 1 & 0 \\
-2 & 1 & 1 & 1 & -1 & 0 & 0 & 0 \\
-1 & 2 & 0 & -1 & 0 & 0 & 0 & 0 \\
-1 & 0 & 2 & -1 & 0 & 0 & 0 & 0 \\
-1 & 0 & 0 & -1 & 2 & 0 & 0 & 0
\end{array}\right) .
\end{aligned}
$$

We can see that the vector in the third row, $\mathbf{p}_{3}^{\prime}$ is a height- 6 vector (sum of three $\mathbf{p}$ vectors), in contrast to the vectors $\mathbf{p}_{i}^{\prime}, i \geq 4$, which are height- 4 vectors (sum of two p-vectors). The condition for faithful compatibility (3.8) requires that $D^{V}$ has rows $\mathbf{r}_{i}$ that can be written as linear combination of vectors $\left\{\mathbf{p}_{3}^{\prime}, \cdots, \mathbf{p}_{n}^{\prime}\right\}$. So there should exist a sequence of row operations on the set $\left\{\mathbf{p}_{i}^{\prime}\right\}$ that transform it to the set $\left\{\mathbf{r}_{i}\right\}$. However, we can see by inspection that there are no elementary row operations that can transform $\mathbf{p}_{3}^{\prime}$ into a height- 4 or height-2 vector. Therefore, there are no $D^{V}$ which is compatible with maximal $D^{Y}$ for $N=4$ and $N=5$.

Now we generalize the previous method to general $N \geq 6$ (the arguments are valid for $N \geq 4$ ). For maximal $D^{Y}$, we need that $\mathbf{q}_{i}$ be distributed into the three types (4.2) as evenly as possible. This is achieved if we assume $\mathbf{q}_{1}, \mathbf{q}_{2}, \mathbf{q}_{3}$ are each of a different type and repeat the subsequent vectors as

$$
\left\{\mathbf{q}_{i}\right\}=\left\{\mathbf{q}_{1}, \mathbf{q}_{2}, \mathbf{q}_{3}, \mathbf{q}_{1}, \mathbf{q}_{2}, \mathbf{q}_{3}, \mathbf{q}_{1}, \cdots\right\} .
$$

Then, we can write $D^{Y}$ in the form (3.7) by subtracting

$$
\begin{aligned}
\mathbf{p}_{3 k+1}^{\prime} & =\mathbf{p}_{3 k+1}-\mathbf{p}_{1}, \quad k \geq 1, \\
\mathbf{p}_{3 k+2}^{\prime} & =\mathbf{p}_{3 k+2}-\mathbf{p}_{2}, \quad k \geq 1, \\
\mathbf{p}_{3 k}^{\prime} & =\mathbf{p}_{3 k}-\mathbf{p}_{3}, \quad k \geq 2,
\end{aligned}
$$

which define all $\mathbf{p}_{i}^{\prime}$ for $i \geq 4$. The remaining $\mathbf{p}_{3}^{\prime}$ is obtained as

$$
\mathbf{p}_{3}^{\prime}=\mathbf{p}_{3}-\mathbf{p}_{1}-\mathbf{p}_{2},
$$

by using the convention that $\mathbf{q}_{3}=\mathbf{q}_{1}+\mathbf{q}_{2}$. We prove the inexistence of $D^{V}$ which is compatible with maximal $D^{Y}$ in two steps: (a) we show that the $\mathbf{p}_{i}^{\prime}$ vectors in (5.16), 
$i \geq 4$, are height- 4 vectors whereas $\mathbf{p}_{3}^{\prime}$ in (5.17) has height 6 ; (b) we demonstrate that the height of $\mathbf{p}_{3}^{\prime}$ can not be reduced by summing or subtracting any $\mathbf{p}_{i}^{\prime}, i \geq 4$, and thus the set $\left\{\mathbf{p}_{i}^{\prime}\right\}, i \geq 3$, cannot be transformed to the set $\left\{\mathbf{r}_{i}\right\}$ by row operations. Step (a) is shown in appendix D while step (b) is detailed in appendix E.

One remark is in order. As explained in ref. [15], constructing $D_{\text {full }}^{Y}$ for the full theory from our reduced $D^{Y}$ may not be feasible for $N \geq 6$. However, such a difficulty is not relevant for the maximal group of order $\left|G_{q}\right|_{\text {bound }}$ which were shown here to be nonrealizable as a discrete symmetry in both sectors. The above difficulty might be relevant for nonmaximal groups of order less than $\left|G_{q}\right|_{\text {bound }}$.

\section{$6 \quad$ Building symmetric models}

The analysis of realizable abelian symmetries of NHDMs restricted to the Yukawa sector and to the Higgs potential separately were given respectively in refs. [15, 39]. In the previous sections, we have seen how to analyze the compatibility between different sectors and have concluded that symmetries of order $\left|G_{q}\right|_{\text {bound }}$ in (3.3) are not realizable in the whole NHDM theory. Here, we discuss methods (A) to construct the maximal number of allowed terms in the Lagrangian from the minimal number of terms, (B) to transfer the symmetry of the Yukawa sector to the potential, (C) to extend the symmetry of the potential to the Yukawa sector and, finally, (D) to construct the full Yukawa terms from the backbone structure.

\subsection{From minimal to maximal number of terms}

In the SNF method, each row in the extracted $D$-matrix corresponds to a Lagrangian term or a pair including the respective hermitean conjugate. From the $D$-matrix, we can straightforwardly analyze the abelian symmetry of the theory. Often, we want to do the opposite: how can we construct the Lagrangian for a given realizable symmetry? In general, an abelian symmetry can be sustained by a minimal number of terms in the Lagrangian and one of the problems is how we extend the minimal terms to the maximal terms allowed by symmetry. The usual method is to take the charges (generators) and explicitly write down all possible terms. Here we present an alternative method of extending a $D$-matrix with the minimal number of rows to a $D$-matrix with the maximal number of compatible rows. This method leads to a unique matrix if the rows of the $D$-matrix (Lagrangian terms) are restricted to certain types and the fields involved are fixed.

We illustrate the method by using one example. Let us take the backbone structure for $\mathbb{Z}_{2} \times \mathbb{Z}_{2}$-3HDM in (4.9) and eliminate some rows until the minimum number of 4 and 2, respectively:

$$
D^{Y}=\left(\begin{array}{ccc|ccc}
1 & -1 & 0 & 1 & -1 & 0 \\
1 & -1 & 0 & -1 & 1 & 0 \\
0 & 1 & -1 & 0 & 1 & -1 \\
0 & 1 & -1 & 0 & -1 & 1
\end{array}\right), \quad D^{V}=\left(\begin{array}{ccc}
2 & -2 & 0 \\
0 & 2 & -2
\end{array}\right)
$$

We can reobtain the matrices in (4.9) by using the following recipe: take all integer linear combinations of the rows that match similar forms. The proposition in section 2.3 ensures 
that this procedure is the necessary and sufficient condition to maintain the symmetry. In our example, the rows of $D^{Y}$ should be of the form $\left(\mathbf{p}_{i}, \mathbf{q}_{i}\right)$ where both $\mathbf{p}_{i}$ and $\mathbf{q}_{i}$ are of the form $(1,-1,0)$ or permuted entries, whereas the rows of $D^{V}$ should have at most height 4 and its components should add to zero. The only linear combinations of the rows of (6.1) that match the criteria are the ones given in (4.9). The rows in (4.9) would give the maximum number of terms in the Lagrangian compatible with the symmetry if their row correspond directly to Lagrangian terms. This is not the case for $D^{Y}$ which is a reduced $D$-matrix. This method of extending the rows of $D$ is quite general and can be applied to any case where a $D$-matrix contains the minimum number of linearly independent rows.

\subsection{From the Yukawa sector to the potential}

We illustrate here a method to transfer the symmetry of the Yukawa terms of NHDMs to the Higgs potential. This method can be adapted to more general cases where the symmetry structure of a sector involving more fields needs to be extended to sectors with less fields, a typical example of the latter being the scalar potential. In this case, the extended $D$-matrix is unique, except for possible ways of rewriting.

We take the $\mathbb{Z}_{2} \times \mathbb{Z}_{2}$-3HDM again, given in (6.1). Suppose we only know the symmetry in the Yukawa sector as in $D^{Y}$ and we want to extend the symmetry to the Higgs potential. To that end, transform $D^{Y}$ to the form (3.7), i.e., eliminate the entries that does not correspond to Higgs fields:

$$
D^{Y}=\left(\begin{array}{ccc|ccc}
1 & -1 & 0 & 1 & -1 & 0 \\
0 & 1 & -1 & 0 & 1 & -1 \\
\hline 2 & -2 & 0 & 0 & 0 & 0 \\
0 & 2 & -2 & 0 & 0 & 0
\end{array}\right)
$$

Take the rows with entries in $Q_{i L}$ eliminated (below the horizontal line) and consider it as $D^{V}$. If $D^{V}$ is sufficient to sustain the discrete symmetry, then we have extracted a faithfully compatible $D^{V}$. This is the case here where each sector sustains a compatible $\mathbb{Z}_{2} \times \mathbb{Z}_{2}$ symmetry, which is the symmetry of the whole theory. Even if the extracted $D^{V}$ is not enough to sustain the discrete symmetry, the symmetry in $D^{V}$ would still be compatible with $D^{Y}$ but additional checks are necessary to see if an accidental U(1) arises after considering all compatible terms.

\subsection{From the potential to the Yukawa sector}

We cover here the remaining case where a discrete symmetry is present in one sector of a theory but it leads to an accidentally continuous symmetry in another sector. In this case the symmetries in the two sectors are compatible but not faithfully compatible as in (3.6). One prime example is the following: a discrete symmetry in the scalar potential but an accidental continuous symmetry in the Yukawa sector. Here we give an example of how to construct theories with such a feature. We do not treat the case $G_{\phi} \subset G_{q}$, with discrete $G_{q}$, as we were unable to find examples.

Our example model is a $\mathbb{Z}_{8}$ symmetric $4 \mathrm{HDM}$. We have seen in section 5 that a $\mathbb{Z}_{8}$ symmetry in the Yukawa sector cannot be compatible to a $\mathbb{Z}_{8}$ symmetry in the Higgs 
potential. However, we still obtain a fine $\mathbb{Z}_{8}$ symmetric model (with regard to the absence of Goldstone bosons) if the Higgs potential exhibits $\mathbb{Z}_{8}$ symmetry but the Yukawa sector has an accidental continuous symmetry containing $\mathbb{Z}_{8}$ as a subgroup.

We begin with the scalar sector symmetric by the desired discrete symmetry and extend such a symmetry to the other sector. A $\mathbb{Z}_{8}$ symmetric Higgs potential is ensured if the phase sensitive (quartic) terms in the potential comes from the $D$-matrix

$$
D^{V}=\left(\begin{array}{cccc}
2 & -1 & -1 & 0 \\
0 & 2 & -1 & -1 \\
0 & 0 & 2 & -2
\end{array}\right) .
$$

This matrix has three rows which is the minimum number to sustain a discrete symmetry for four fields $\phi_{i}$, with one automatic U(1) symmetry. In this case, there are no other phase-sensitive terms except their hermitean conjugates.

The next step is to construct the reduced matrix $D^{Y}$ for the Yukawa sector which is compatible with $D^{V}$ in (6.3). We need 5 linearly independent rows to sustain a discrete symmetry for 7 fields $\left(\phi_{i}, Q_{i L}\right)$, with two automatic $\mathrm{U}(1)$ symmetries. Therefore, four linearly independent rows are sufficient to sustain an accidental U(1) and no more. Given that each row of $D^{Y}$ should have a well defined form, we can construct from the first two rows of $D^{V}$,

$$
D^{Y}=\left(\begin{array}{cccc|ccc}
1 & -1 & 0 & 0 & 1 & -1 & 0 \\
1 & 0 & -1 & 0 & -1 & 1 & 0 \\
0 & 1 & -1 & 0 & 0 & 1 & -1 \\
0 & 1 & 0 & -1 & 0 & -1 & 1
\end{array}\right)
$$

We can see that by summing the second row to the first and the fourth row to the third we effectively obtain $D^{V}$ in the first and third rows and there is no other way we can eliminate the entries in the $Q_{L}$-sector. As other choices - picking two other rows of $D^{V}$ or relabeling $Q_{i L}$ fields - are possible, we can see that this procedure does not lead to unique extensions. Nevertheless, the matrix $D_{\text {full }}^{Y}$, describing the actual Yukawa terms, can be obtained from the procedure outlined in section 6.4.

When we consider $N$ Higgs doublets, with $N>4$, we need $D^{Y}$ to have $N$ linearly independent rows to allow only one additional U(1) symmetry. The first four rows of $D^{Y}$ can always be chosen as in (6.4) by using two different $\mathbf{q}$-vectors as, for example, $\mathbf{q}_{1}=(1,-1,0)$ and $\mathbf{q}_{3}=(0,1,-1)$ in (6.4). However, additional linearly independent rows cannot be added at will because any additional $\mathbf{q}_{i}, i \geq 5$, will be linearly dependent to $\mathbf{q}_{1}$ and $\mathbf{q}_{3}$. If more compatible linearly independent rows can not be added, then more additional $\mathrm{U}(1)$ symmetries will be present in the Yukawa sector. This is usually the case for large symmetries in $D^{V}$ and $N>4$. If additional compatible rows can be added in sufficient number, then only one accidental U(1) may be present. The checking may be performed by using the discrete charges of the Higgs doublets.

\subsection{From reduced $D^{Y}$ to full $D^{Y}$}

We present here a method to construct the full $D_{\text {full }}^{Y}$, involving all fields of the Yukawa terms $\left(\phi_{a}, Q_{i L}, d_{i R}, u_{i R}\right)$, in terms of the reduced $D^{Y}$, involving only $\left(\phi_{a}, Q_{i L}\right)$ (also refereed to as 
a backbone structure). This is an alternative method to the one presented in ref. [15], where charges where used to reconstruct compatible Yukawa terms. The method is guaranteed to work for $N=2,3,4,5$ but it is more interesting for $N>2$ so that we focus on those cases. The extended $D$-matrix is not unique in general.

We illustrate the method by constructing the Yukawa interactions of the $\mathbb{Z}_{4}$ symmetric $3 \mathrm{HDM}$ from the backbone structure (4.7). From the potential part, $D^{V}$, we extract the two phase sensitive terms in the potential and complete with hermitean terms. From the Yukawa part, $D^{Y}$, we can construct Yukawa terms. We first take $D^{Y}$, with the minimal number of rows

$$
D^{Y}=\left(\begin{array}{ccc|ccc}
1 & -1 & 0 & 1 & -1 & 0 \\
1 & -1 & 0 & -1 & 1 & 0 \\
0 & 1 & -1 & 0 & 1 & -1 \\
1 & 0 & -1 & 0 & -1 & 1
\end{array}\right)
$$

For $N=3$, we have $n=N+1=4$ rows in the minimal $D^{Y}$. We should associate to each right-handed quark $d_{i R}$ and $u_{i R}$ a row of $D^{Y}$ so that all rows are exhausted. For concreteness, we associate

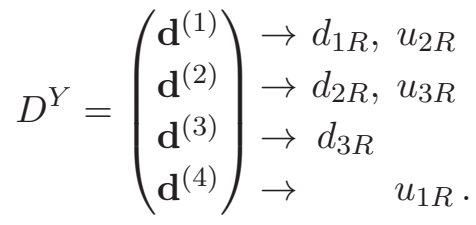

For $N=3,4$, some rows should be simultaneously associated to more than one righthanded field. For $N=5$, exactly one right-handed field is paired up with exactly one row. For $N \geq 6$, one right-handed field should be linked to more than one row and more constraints emerge.

Next, we follow each link in (6.6) and build a pair of $D$-matrix vectors (rows). For example, for $\mathbf{d}^{(1)} \rightarrow d_{1 R}$ we construct two pairs of vectors,

$$
\mathbf{d}^{(1)} \rightarrow\left\{\begin{array}{l}
\mathbf{u}^{(1)}\left(d_{1 R}\right)=\left(e_{1} ;-e_{2} ; e_{1} ; 0_{3}\right) \\
\mathbf{u}^{\left(1^{\prime}\right)}\left(d_{1 R}\right)=\left(e_{2} ;-e_{1} ; e_{1} ; 0_{3}\right)
\end{array},\right.
$$

where we use the compact notation of canonical vectors $\left(e_{i}\right)_{j}=\delta_{i j}$ and follow the ordering $\left(\phi_{a} ; Q_{i L} ; d_{i R} ; u_{i R}\right)$. The first vector, for example, reconstructs the Yukawa term $\bar{Q}_{2 L} \phi_{1} d_{1 R}$, while the second reconstructs $\bar{Q}_{1 L} \phi_{2} d_{1 R}$. These two terms are the only ones involving $d_{1 R}$ that satisfies $\mathbf{u}^{(1)}\left(d_{1}\right)-\mathbf{u}^{\left(1^{\prime}\right)}\left(d_{1}\right)=\mathbf{d}^{(1)}$. Since the Yukawa terms for up-type quarks are different, the vectors for them are different. For example, for $\mathbf{d}^{(1)} \rightarrow u_{2 R}$ we construct two pairs of vectors,

$$
\mathbf{d}^{(1)} \rightarrow\left\{\begin{array}{l}
\mathbf{u}^{(1)}\left(u_{2 R}\right)=\left(-e_{1} ;-e_{1} ; 0_{3} ; e_{2}\right) \\
\mathbf{u}^{\left(1^{\prime}\right)}\left(u_{2 R}\right)=\left(-e_{2} ;-e_{2} ; 0_{3} ; e_{2}\right)
\end{array},\right.
$$

obeying $\mathbf{u}^{(1)}\left(u_{2 R}\right)-\mathbf{u}^{\left(1^{\prime}\right)}\left(u_{2 R}\right)=-\mathbf{d}^{(1)}$. The two vectors correspond unambiguously to the Yukawa terms $\bar{Q}_{2 L} \tilde{\phi}_{2} u_{2 R}$ and $\bar{Q}_{1 L} \tilde{\phi}_{1} u_{2 R}$. 
By applying the recipe to all associations in (6.6), following the order $\left(d_{1 R}, d_{2 R}, d_{3 R}, u_{1 R}, u_{2 R}, u_{3 R}\right)$, we obtain the $12 \times n_{F}$ matrix

$$
D_{\text {full }}^{Y}=\left(\begin{array}{ccc|ccc|ccc|ccc}
1 & 0 & 0 & 0 & -1 & 0 & 1 & 0 & 0 & 0 & 0 & 0 \\
0 & 1 & 0 & -1 & 0 & 0 & 1 & 0 & 0 & 0 & 0 & 0 \\
1 & 0 & 0 & -1 & 0 & 0 & 0 & 1 & 0 & 0 & 0 & 0 \\
0 & 1 & 0 & 0 & -1 & 0 & 0 & 1 & 0 & 0 & 0 & 0 \\
0 & 1 & 0 & 0 & 0 & -1 & 0 & 0 & 1 & 0 & 0 & 0 \\
0 & 0 & 1 & 0 & -1 & 0 & 0 & 0 & 1 & 0 & 0 & 0 \\
\hline-1 & 0 & 0 & 0 & 0 & -1 & 0 & 0 & 0 & 1 & 0 & 0 \\
0 & 0 & -1 & 0 & -1 & 0 & 0 & 0 & 0 & 1 & 0 & 0 \\
-1 & 0 & 0 & -1 & 0 & 0 & 0 & 0 & 0 & 0 & 1 & 0 \\
0 & -1 & 0 & 0 & -1 & 0 & 0 & 0 & 0 & 0 & 1 & 0 \\
-1 & 0 & 0 & 0 & -1 & 0 & 0 & 0 & 0 & 0 & 0 & 1 \\
0 & -1 & 0 & -1 & 0 & 0 & 0 & 0 & 0 & 0 & 0 & 1
\end{array}\right) .
$$

The vectors constructed in (6.7) and (6.8) correspond to the rows 1,2,9,10, respectively. One can check this matrix still exhibits $\mathbb{Z}_{4}$ symmetry.

We should note that care must be taken when extracting the symmetries of subsectors. If we take the first (last) 6 rows of (6.9) associated to the $d_{R^{-} \text {-sector }}\left(u_{R^{-}}\right.$-sector) and compute its SNF, we conclude that it exhibits a $\mathbb{Z}_{2} \times \mathrm{U}(1)\left[\mathbb{Z}_{2} \times \mathrm{U}(1)\right]$ symmetry. This could indicate that the $\mathrm{d}$ - and $\mathrm{u}$-sectors possess each an accidentally larger symmetry which intersects into the $\mathbb{Z}_{4}$ symmetry when both sectors are considered. That is not the case as we can compute the $\mathbb{Z}_{4}$ charges in the entire theory and list all the compatible Yukawa terms. We discover that in each sector there is one term missing which reduces the apparent larger symmetry to $\mathbb{Z}_{4}$ in each sector; see more details in appendix F. Therefore, it is very important to consider all compatible terms (rows) when analyzing the symmetries of subsectors. In general, subsectors may or may not possess larger accidental symmetries. For example, in appendix $\mathrm{F}$, we show another $\mathbb{Z}_{4}-3 \mathrm{HDM}$, constructed from the same backbone structure (4.7), possessing an accidental U(1) symmetry in the $u_{R}$-sector. These examples also illustrate the fact that the construction procedure (6.6) does not lead to a unique $D_{\text {full }}^{Y}$.

A remark considering the proposition of section 2.3 is in order. The matrix $D_{\text {full }}^{Y}$ in (6.9) may present an apparent contradiction to the proposition as one should be able to write the additional d-type row as a linear combination of the 12 rows of $D_{\text {full }}^{Y}$ but, as the SNF factors change, it cannot be written as a linear combination of the first 6 rows (d-type) alone. Both applications of the proposition are correct and the point is that both $\mathrm{d}$ - and u-type rows of $D_{\text {full }}^{Y}$ need to be combined to write the additional d-type row. Therefore, the recipe in section 6.1 is still valid but one must be careful when using fewer rows (restricting to subsectors).

A similar procedure can be applied to backbone structures for $N=4,5$. Easily identifiable variants can be constructed by relabeling equal-type fields. Genuinely different models (different symmetries) can be constructed by choosing different rows of the reduced $D^{Y}$; see example in appendix $\mathrm{F}$. 


\begin{tabular}{|c|ccccc|}
\hline$N$ & 2 & 3 & 4 & 5 & 6 \\
\hline$\left|G_{q}\right| \leq$ & 3 & 5 & 8 & 12 & 16 \\
$\left|G_{\phi}\right| \leq$ & 2 & 4 & 8 & 16 & 32 \\
$\left|G_{F}\right| \leq$ & 2 & 4 & $\underline{8}$ & $\underline{12}$ & $\underline{16}$ \\
\hline
\end{tabular}

Table 1. Bound for the order of abelian symmetry $G_{F}=G_{q}=G_{\phi}$ acting compatibly on full $N$-HDM. $G_{q}$ and $G_{\phi}$ denote the symmetries in the Yukawa and Higgs potential, respectively.

\section{Discussion and conclusion}

In this work we have presented techniques to analyze the abelian symmetries in full models taking the class of general N-Higgs-doublet models as an example. The techniques are based on the Smith normal form and extends the results of our previous work [15] by focusing on methods to analyze the compatibility between abelian symmetries acting in two different sectors - the Yukawa sector and the Higgs potential in our case. Application to other full models containing two or more sectors follows analogously. We have also presented techniques to construct symmetric models by extending the symmetry from one sector to another.

The main result within N-Higgs-doublet models is an updated list of abelian symmetries that acts compatibly (realizable) in both Yukawa interactions and Higgs potential. We focus in faithfully compatible symmetries where the same symmetry acts faithfully in both sectors and no larger symmetry is accidentally present in either sector. As a result, we have concluded that realizable abelian symmetries $G_{F}$ in the full NHDM should obey the bound

$$
\left|G_{F}\right|< \begin{cases}n^{2} / 3, & \text { if } n \text { is divisible by } 3, \\ \left(n^{2}-1\right) / 3, & \text { if } n \text { is not divisible by } 3,\end{cases}
$$

where $n=N+1$. Table 1 summarizes our results for small $N$. The underlined numbers mean that the order of the group should be strictly smaller. In special, $\mathbb{Z}_{2}-2 \mathrm{HDM}$ is long known to be constructible whereas the $\mathbb{Z}_{2} \times \mathbb{Z}_{2}-3$ HDM and $\mathbb{Z}_{4}-3$ HDM were explicitly shown to be realizable in section 4 . We should note that $\mathbb{Z}_{2} \times \mathbb{Z}_{2}-3$ HDM models presented here are different from the Weinberg model [17] in the Yukawa sector as the charges are not flavor universal.

If we allow for accidental symmetries, then the bound (7.1) does not need to be respected. In special, $\mathbb{Z}_{8}-4 \mathrm{HDM}$ is possible: the theory possesses a $\mathbb{Z}_{8}$ symmetry in the Higgs potential but an accidental U(1) symmetry in the Yukawa sector. Usually, more Higgs doublets and large abelian symmetries in the Higgs sector leads to more accidental $\mathrm{U}(1)$ symmetries in the Yukawa sector.

Based on the results presented here, we can also draw several conclusions about the supersymmetric case, i.e., the supersymmetric version of NHDMs with $N$ pairs of Higgs doublets $H_{u i}, H_{d i}$, with $i=1, \ldots, N$; we denote them as $2 N$-HMSSM. To use our results, we rename $\phi_{i}=H_{d i}$ and $\phi_{N+i}=\epsilon H_{u i}^{*}$. The immediate restrictions are as follows: 
- The Higgs potential cannot realize any discrete rephasing symmetry $\mathbb{Z}_{n}$, i.e., either the symmetry is softly broken or it is realized as a continuous accidental U(1) symmetry. This follows because the only phase sensitive terms in the potential come from soft-breaking quadratic terms $\phi_{i}^{\dagger} \phi_{j}$ which contribute as height 2 vectors in the $D$-matrix and this kind of matrix does not lead to any discrete symmetry (see lemma in ref. [15]).

- The bound $\left|G_{q}\right|_{\text {bound }}$ in eq. (3.3) now applies separately to the down-type ( $\left.\bar{d} y_{i}^{d} Q H_{d i}\right)$ and up-type $\left(\bar{u} y_{i}^{u} Q H_{u i}\right)$ Yukawa interactions (superpotential) since each sector involves separate Higgs (super) fields. Within each sector, there are two automatic symmetries corresponding to hypercharge and baryon number. In the whole Yukawa interactions, a combination of previous symmetries leads to an additional automatic U(1) corresponding to Peccei-Quinn symmetry. There are two reduced matrices (3.5) now, one for each sector, and they can be made compatible if the charges for $Q$ are compatible. So the MSSM cannot sustain any discrete symmetry while $\mathbb{Z}_{3}$ is the maximal abelian symmetry that is realizable in the Yukawa sector of the extension of MSSM with four Higgs doublets (4-HMSSM), provided that $\mathbb{Z}_{3}$ is realizable within down- or up-type sector.

- The previous bound on the symmetry of Yukawa interactions can be evaded if we allow for an additional accidental U(1) symmetry in both down- and up-sectors, so that their intersection leads to a discrete symmetry. For example, $\mathbb{Z}_{4}$ and $\mathbb{Z}_{2} \times \mathbb{Z}_{2}$ are realizable in 4 -HMSSM and they are the maximal groups. For general $2 N$-HMSSM, we can show that the symmetry group consists of at most two factors $G_{q}=\mathbb{Z}_{k_{1}} \times \mathbb{Z}_{k_{2}}$ $\left(k_{1}\right.$ divides $k_{2}$ ) and an example with $G_{q}=\mathbb{Z}_{N} \times \mathbb{Z}_{N}$ or $\mathbb{Z}_{N^{2}}$ can be readily constructed. Therefore a group of order $N^{2}$, which is always larger than the bound $\left|G_{q}\right|_{\text {bound }}$ in eq. (3.3), is always realizable but its maximality remains to be checked.

In summary, the techniques presented here were shown to be powerful to analyze the possible abelian symmetries of full N-Higgs-doublet models (and its supersymmetric extensions). Its capabilities, however, are not restricted to these classes of models and further application can be considered on other contexts where discrete or continuous symmetries are crucial. Recent examples can be found in the active areas of neutrino flavor, dark matter and axion model building.

\section{Acknowledgments}

The author thanks Igor Ivanov and Rabindra Mohapatra for very helpful comments. This work was partially supported by $\mathrm{CNPq}$, Conselho Nacional de Desenvolvimento Científico e Tecnológico - Brasil, and by Brazilian Fapesp through grants 2013/26371-5 and 2013/22079-8. 


\section{A Proof of proposition}

Let us prove the main proposition of section 2.3. Suppose $D$ has size $n \times m$ and rank $n$. We denote by $D^{\prime}$ the matrix $D$ after addition of the row $\mathbf{u}=\left(u_{1}, u_{2}, \cdots, u_{m}\right)$ :

$$
D^{\prime}=\left(\frac{D}{\mathbf{u}}\right)
$$

The existence of $\mathrm{SNF}$ for $D, R D C=\mathrm{SNF}(D)$, implies

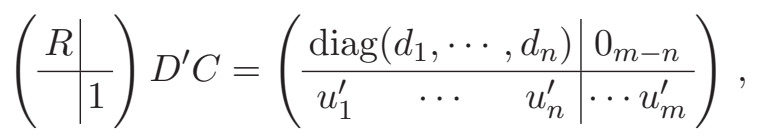

where $u_{i}^{\prime}=(\mathbf{u} C)_{i}$. Firstly, $u_{1}^{\prime}$ should be divisible by $d_{1}$ because otherwise we could write

$$
u_{1}^{\prime}=k_{1} d_{1}+r_{1}, \quad 0<r_{1}<d_{1},
$$

and then we could replace $u_{1}^{\prime}$ by its remainder $r_{1}$ after subtracting a multiple of the first row from the last row. If $r_{1}$ divides $d_{1}$, then $r_{1}$ would be the first factor of the SNF of $D^{\prime}$ which is a contradiction. If $r_{1}$ does not divide $d_{1}$, then a even smaller number could be produced by subtraction and consequently leading to a factor in $\operatorname{SNF}\left(D^{\prime}\right)$ smaller than $d_{1}$ which is a contradiction as well. From similar arguments, all $u_{i}^{\prime}, i>1$, should be divisible by $d_{1}$. We are then left with

$$
D^{\prime} \sim\left(\begin{array}{c|c}
d_{1} & 0 \cdots 0 \\
\hline 0 & \\
\vdots & B \\
0 &
\end{array}\right)
$$

where $B$ has entries divisible by $d_{1}$ in the same structure as (A.2), without the first row and column. Now, the same arguments as above apply to $B$ and the second factor $d_{2}$. We can continue until the $d_{n}$ factor and then all $u_{i}^{\prime}, 1 \leq i \leq n$, can be eliminated by row operations. The remaining $u_{i}^{\prime}, i>n$, should be zero because otherwise there would be more nonzero factors in $\operatorname{SNF}\left(D^{\prime}\right)$.

We conclude that $\mathbf{u}^{\prime}=\mathbf{u} C$ is a linear combination of the rows of (A.2) which implies $\mathbf{u}$ is a linear combination of the rows of $D$.

Finally, all the arguments above remain valid if the rank of $D$ is smaller than its number of rows $n$.

\section{B Rephasing space}

Here we clarify the distinction between the groups $\left\{\tilde{G}_{q}, \tilde{G}_{\phi}\right\}$ and $\left\{G_{q}, G_{\phi}\right\}$, and stress that the former enter in $G_{F}=\tilde{G}_{q} \cap \tilde{G}_{\phi}$. The notation $\tilde{G}$ refers to the symmetry group considered as a subgroup of $[\mathrm{U}(1)]^{n_{F}}$, the rephasing symmetry of all $n_{F}$ fields involved. We present simple examples below where the distinction is important. In the text, we have disregarded automatic symmetries in $G_{q}$ and $G_{\phi}$. 
Let us adopt the following notation: we consider a theory consisting of two sectors described by Lagrangians $\mathscr{L}_{1,2}$, with symmetries denoted either as $G_{1,2}$ when restricted to lagrangians $\mathscr{L}_{1,2}$ or as $\tilde{G}_{1,2}$ when considering all fields appearing in the whole theory $\left(\mathscr{L}_{1}+\mathscr{L}_{2}\right)$.

For the first example, consider two noninteracting complex scalar fields $\varphi_{1,2}$ and a theory where the only phase sensitive terms are

$$
\mathscr{L}_{1}=\varphi_{1}^{4}, \quad \mathscr{L}_{2}=\varphi_{2}^{3}
$$

We have suppressed coupling coefficients and hermitean conjugates for simplicity. It is clear that $\mathscr{L}_{1}\left(\mathscr{L}_{2}\right)$ sustains a $\mathbb{Z}_{4}\left(\mathbb{Z}_{3}\right)$ symmetry when restricted to $\varphi_{1}\left(\varphi_{2}\right)$ sector so that $G_{1}=\mathbb{Z}_{4}$ $\left(G_{2}=\mathbb{Z}_{3}\right)$. However, to analyze the compatibility of symmetries (section 3 ) and extract the common symmetry of the whole theory, $G_{F}$, we need to consider $\tilde{G}_{1}=\mathbb{Z}_{4} \times \mathrm{U}(1)_{2}$ and $\tilde{G}_{2}=\mathrm{U}(1)_{1} \times \mathbb{Z}_{3}$ which are subgroups of the whole rephasing group $[\mathrm{U}(1)]^{2}=\mathrm{U}(1)_{1} \times \mathrm{U}(1)_{2}$ for two fields. Clearly $G_{F}=\tilde{G}_{1} \cap \tilde{G}_{2}=\mathbb{Z}_{4} \times \mathbb{Z}_{3}$, although $G_{1} \cap G_{2}=\{e\}$ abstractly. This situation can be easily detected in the $D$-matrix as it would be separable into blocks.

For the second example, we consider a theory with the presence of an automatic discrete symmetry. We consider two chiral fermion fields $\psi_{1,2}$ and one complex scalar field $\varphi$. The two sectors consist of phase sensitive terms

$$
\begin{aligned}
-\mathscr{L}_{1} & =\overline{\psi_{1}^{c}} \psi_{1}+\overline{\psi_{1}^{c}} \psi_{2} \varphi+\overline{\psi_{2}^{c}} \psi_{2} \varphi^{*}, \\
\mathscr{L}_{2} & =\varphi^{3}
\end{aligned}
$$

We can see this theory is invariant by $G_{F}=\mathbb{Z}_{6}$ symmetry:

$$
\psi_{1} \rightarrow-\psi_{1}, \quad \psi_{2} \rightarrow e^{i 2 \pi / 6} \psi_{2}, \quad \varphi \rightarrow e^{i 2 \pi / 3} \varphi
$$

However, the scalar sector is only sensitive to the $\mathbb{Z}_{3}$ subgroup of $\mathbb{Z}_{6}$. The $\mathbb{Z}_{2}$ subgroup is automatic and it is a discrete version of fermion number: $\psi_{1,2}$ are odd whereas $\varphi$ is even. The symmetry $\mathbb{Z}_{6}$ is the intersection of $\tilde{G}_{2}=\mathbb{Z}_{3} \times[\mathrm{U}(1)]^{2}$ and $\tilde{G}_{1}=G_{1}=\mathbb{Z}_{6}$, although clearly $G_{2}=\mathbb{Z}_{3}$. In the SNF method, the information on the automatic $\mathbb{Z}_{2}$ can be seen on the $\mathbb{Z}_{6}$-charge,

$$
\mathbf{s}=(3,1,2),
$$

with ordering $\left(\psi_{1}, \psi_{2}, \varphi\right)$, as the scalar charge has a factor that divides $\left|G_{F}\right|=6$. The same happens if the charges for a set of fields (e.g. scalars) has a common factor $k$ which divides the whole group. See more examples of models with automatic discrete lepton number in ref. [14]. In particular, a type-II seesaw model presents $G_{1}=\mathbb{Z}_{3}$ and $G_{2}=\mathbb{Z}_{3}$ but $G_{F}=\mathbb{Z}_{6}$.

\section{Backbone structure for maximal symmetry in 3HDM}

Let us show that the backbone structures shown in (4.7) and (4.9), corresponding to the order 4 abelian symmetries $\mathbb{Z}_{4}$ and $\mathbb{Z}_{2} \times \mathbb{Z}_{2}$ respectively, are unique up to reordering of $\phi_{a}$ and $Q_{i L}$ fields. 
The minimal $D^{Y}$ matrix for $3 \mathrm{HDM}$ has the form in (4.1). In this case, the $\mathbf{p}$ - and $\mathbf{q}-$ vectors are any of the six vectors that can be categorized into the three types of nonparallel vectors in eq. (4.2). We have seen in [15] that $\left|G_{q}\right|$ is bounded above by $m=n_{1} n_{2}+n_{2} n_{3}+$ $n_{3} n_{1}$ where $n_{i}$ is the number of $i$-th type $\mathbf{q}$-vector (or, in this case, $\mathbf{p}$-vector as well). To get $\left|G_{q}\right|=4$, we can allow $\left(n_{1} n_{2} n_{3}\right)=(211)$ or $\left(n_{1} n_{2} n_{3}\right)=(220)$. Therefore, we have at least one and at most two q-vectors (p-vectors) parallel (of the same type).

We know that the order of the abelian group, $\left|G_{F}\right|$, can be given by the modulus of a determinant-like $E$-function which is a function of the rows of the $D$-matrix [15]. Such function can be expanded as

$$
E\left(D^{Y}\right)=E\left(\mathbf{p}_{1}+\mathbf{q}_{1}, \cdots, \mathbf{p}_{4}+\mathbf{q}_{4}\right)=(1 \not 234)-(12231)+(1 \not 221)-(1 \not 224)-(\not 214),
$$

where we use the shorthand: $\mathbf{p}_{i} \rightarrow i, \mathbf{q}_{k} \rightarrow \not k$ and suppress the $E$ function so that the first term represent $E\left(\mathbf{q}_{1}, \mathbf{q}_{2}, \mathbf{p}_{3}, \mathbf{p}_{4}\right)$. We also use determinant-like properties for $E$; see ref. [15] for more details. We also conventionally choose $\mathbf{q}_{4}=\mathbf{q}_{1}$ to be the parallel vectors. And then $\mathbf{q}_{2}$ and $\mathbf{q}_{3}$ cannot be parallel to $\mathbf{q}_{1}$. We are left with two possibilities: either $\mathbf{q}_{3}$ is parallel to $\mathbf{q}_{2}$ or not. To avoid cancellations in (C.1), we need to impose $\mathbf{p}_{4} \neq \mathbf{p}_{1}$ as well. From the properties of $E$, one can also check that exchanging $\mathbf{p}_{1} \leftrightarrow \mathbf{p}_{4}$ only amounts to an overall sign change.

Recall that any of the five terms in (C.1) is \pm 1 or 0 , the latter being possible only if some of the two p-vectors or q-vectors are parallel. To get $\left|E\left(D^{Y}\right)\right|=4$, we need exactly one vanishing term. Each of the first four terms only vanish if its $\mathbf{p}$-vectors are parallel. The last term in (C.1) vanishes if one (or both) of the pairs $\left\{\mathbf{q}_{2}, \mathbf{q}_{3}\right\}$ or $\left\{\mathbf{p}_{1}, \mathbf{p}_{4}\right\}$ is parallel. In the first case, we can conventionally adopt $\mathbf{q}_{3}=\mathbf{q}_{2}$ and, among the $\mathbf{p}$-vectors, only $\left\{\mathbf{p}_{1}, \mathbf{p}_{4}\right\}$ or $\left\{\mathbf{p}_{2}, \mathbf{p}_{3}\right\}$ may be parallel to avoid the first four terms from vanishing. However, since $\mathbf{q}_{4}=\mathbf{q}_{1}$ and $\mathbf{q}_{3}=\mathbf{q}_{2}$, parallel $\left\{\mathbf{p}_{2}, \mathbf{p}_{3}\right\}$ is equivalent to parallel $\left\{\mathbf{p}_{1}, \mathbf{p}_{4}\right\}$ after we exchange $\mathbf{p}_{2} \leftrightarrow \mathbf{p}_{1}$ and $\mathbf{p}_{3} \leftrightarrow \mathbf{p}_{4}$.

If $\mathbf{q}_{3}, \mathbf{q}_{2}$ are not parallel, then we must choose some other pair of $\mathbf{p}$-vectors to be parallel. We have three possibilities: $\mathbf{p}_{2}= \pm \mathbf{p}_{1}, \mathbf{p}_{3}= \pm \mathbf{p}_{1}, \mathbf{p}_{4}= \pm \mathbf{p}_{1}$. The possibility that $\mathbf{p}_{2}$ or $\mathbf{p}_{3}$ is parallel to $\mathbf{p}_{4}$ is taken into account from the exchange symmetry $\mathbf{p}_{1} \leftrightarrow \mathbf{p}_{4}$. The possibility $\mathbf{p}_{3}= \pm \mathbf{p}_{1}$ can be also taken into account by exchanging the second and third row of $D^{Y}$, i.e., $\mathbf{q}_{2} \leftrightarrow \mathbf{q}_{3}$ and $\mathbf{p}_{2} \leftrightarrow \mathbf{p}_{3}$.

Summarizing all possibilities, we are left with the following cases

$$
\begin{aligned}
& \text { (A) } \mathbf{q}_{3}=\mathbf{q}_{2} \text { and } \mathbf{p}_{4}= \pm \mathbf{p}_{1} \\
& \text { (B) }\left\{\mathbf{q}_{2}, \mathbf{q}_{3}\right\} \text { nonparallel and } \mathbf{p}_{2}= \pm \mathbf{p}_{1} \\
& \text { (C) }\left\{\mathbf{q}_{2}, \mathbf{q}_{3}\right\} \text { nonparallel and } \mathbf{p}_{4}= \pm \mathbf{p}_{1}
\end{aligned}
$$

We analyze them in detail in the following.

For case (A), the expansion of (C.1) becomes

$$
\begin{aligned}
E\left(D^{Y}\right) & = \pm(1231)-(12231)+(1 \not 221) \mp(12221) \\
& =-2(12231)+2(1222)
\end{aligned}
$$


where we chose $\mathbf{p}_{4}=-\mathbf{p}_{1}$ in the second line to avoid cancellations. Further cancellation is avoided only if $D^{Y}$ has one of the forms

$$
D_{A .1}^{Y}=\left(\begin{array}{c|c}
\mathbf{p}_{1} & \mathbf{q}_{1} \\
\mathbf{p}_{2} & \mathbf{q}_{2} \\
-\mathbf{p}_{2} & \mathbf{q}_{2} \\
-\mathbf{p}_{1} & \mathbf{q}_{1}
\end{array}\right) \quad \text { or } D_{A .2}^{Y}=\left(\begin{array}{c|c}
\mathbf{p}_{1} & \mathbf{q}_{1} \\
\mathbf{p}_{2} & \mathbf{q}_{2} \\
-\mathbf{p}_{2} \pm \mathbf{p}_{1} & \mathbf{q}_{2} \\
-\mathbf{p}_{1} & \mathbf{q}_{1}
\end{array}\right)
$$

where the sign in the last case should be chosen so as to make $\mathbf{p}_{3}=-\mathbf{p}_{2} \pm \mathbf{p}_{1}$ a $\mathbf{p}$-vector.

For case (B), we expand $\mathbf{q}_{3}=\mathbf{q}_{1}+\mathbf{q}_{2}$ by choosing the signs appropriately. The expansion of (C.1) reads

$$
\begin{aligned}
E\left(D^{Y}\right) & =(1234)-(1231) \mp(1214)-(1214), \\
& =(12234)+(1213)-2(1214),
\end{aligned}
$$

where we chose $\mathbf{p}_{2}=\mathbf{p}_{1}$ in the second line to avoid cancellations. There is no cancellation if $\mathbf{p}_{3}=-\mathbf{p}_{4}-\mathbf{p}_{1}$ and we get

$$
D_{B}^{Y}=\left(\begin{array}{c|c}
\mathbf{p}_{1} & \mathbf{q}_{1} \\
\mathbf{p}_{1} & \mathbf{q}_{2} \\
-\mathbf{p}_{1}-\mathbf{p}_{4} & \mathbf{q}_{1}+\mathbf{q}_{2} \\
\mathbf{p}_{4} & \mathbf{q}_{1}
\end{array}\right)
$$

Note that $\mathbf{p}_{3}=-\mathbf{p}_{4}-\mathbf{p}_{1}$ should be a $\mathbf{p}$-vector.

For case (C), we expand again $\mathbf{q}_{3}=\mathbf{q}_{1}+\mathbf{q}_{2}$. The expansion of (C.1) reads

$$
\begin{aligned}
E\left(D^{Y}\right) & = \pm(1231)-(1231)+(1221) \mp(1221), \\
& =-2(1231)+2(1221),
\end{aligned}
$$

where we need to choose $\mathbf{p}_{4}=-\mathbf{p}_{1}$ in the second line. The necessary form for $\mathbf{p}_{3}$ can be extracted and we obtain

$$
D_{C .1}^{Y}=\left(\begin{array}{c|c}
\mathbf{p}_{1} & \mathbf{q}_{1} \\
\mathbf{p}_{2} & \mathbf{q}_{2} \\
-\mathbf{p}_{2} & \mathbf{q}_{1}+\mathbf{q}_{2} \\
-\mathbf{p}_{1} & \mathbf{q}_{1}
\end{array}\right) \text { or } D_{C .2}^{Y}=\left(\begin{array}{c|c}
\mathbf{p}_{1} & \mathbf{q}_{1} \\
\mathbf{p}_{2} & \mathbf{q}_{2} \\
-\mathbf{p}_{2} \pm \mathbf{p}_{1} & \mathbf{q}_{1}+\mathbf{q}_{2} \\
-\mathbf{p}_{1} & \mathbf{q}_{1}
\end{array}\right)
$$

where all rows should correspond to allowed $\mathbf{p}$ - and $\mathbf{q}$-vectors.

By choosing $\mathbf{p}_{1}=\mathbf{q}_{1}=(1,-1,0)$ and $\mathbf{p}_{2}=\mathbf{q}_{2}=(0,1,-1)$ we can rewrite (C.4) and (C.8) as

$$
D_{A .2}^{Y}=\left(\begin{array}{ccc|ccc}
1 & -1 & 0 & 1 & -1 & 0 \\
0 & 1 & -1 & 0 & 1 & -1 \\
-1 & 0 & 1 & 0 & 1 & -1 \\
-1 & 1 & 0 & 1 & -1 & 0
\end{array}\right), \quad D_{C .1}^{Y}=\left(\begin{array}{cccccc}
1 & -1 & 0 & 1 & -1 & 0 \\
0 & 1 & -1 & 0 & 1 & -1 \\
0 & -1 & 1 & 1 & 0 & -1 \\
-1 & 1 & 0 & 1 & -1 & 0
\end{array}\right)
$$


and

$$
D_{A .1}^{Y}=\left(\begin{array}{cccccc}
1 & -1 & 0 & 1 & -1 & 0 \\
0 & 1 & -1 & 0 & 1 & -1 \\
0 & -1 & 1 & 0 & 1 & -1 \\
-1 & 1 & 0 & 1 & -1 & 0
\end{array}\right), D_{C .2}^{Y}=\left(\begin{array}{cccccc}
1 & -1 & 0 & 1 & -1 & 0 \\
0 & 1 & -1 & 0 & 1 & -1 \\
-1 & 0 & 1 & 1 & 0 & -1 \\
-1 & 1 & 0 & 1 & -1 & 0
\end{array}\right)
$$

We can see they all have three rows in common. In fact the matrices in (C.9) are compatible with the $\mathbb{Z}_{4}$ charges in (4.8), i.e., upon multiplication on the charges, it gives 4 times a vector of relatively prime integers. Analogously, the matrices in (C.9) are compatible with the $\mathbb{Z}_{2} \times \mathbb{Z}_{2}$ charges in (4.10).

For case (B), by choosing $\mathbf{p}_{1}=\mathbf{q}_{1}=(1,-1,0)$ and $\mathbf{q}_{2}=\mathbf{p}_{4}=(0,1,-1)$, eq. (C.5) reads

$$
D_{B}^{Y}=\left(\begin{array}{cccccc}
1 & -1 & 0 & 1 & -1 & 0 \\
1 & -1 & 0 & 0 & 1 & -1 \\
-1 & 0 & 1 & 1 & 0 & -1 \\
0 & 1 & -1 & 1 & -1 & 0
\end{array}\right)
$$

The SNF form of this matrix reveals a $\mathbb{Z}_{4}$ symmetry which is not compatible with the matrices in (C.9). But if we choose $\mathbf{p}_{1}=\mathbf{q}_{1}=(1,0,-1)$ and $\mathbf{q}_{2}=\mathbf{p}_{4}=(0,-1,1)$, we obtain

$$
D_{B}^{Y}=\left(\begin{array}{cccccc}
1 & 0 & -1 & 1 & 0 & -1 \\
1 & 0 & -1 & 0 & -1 & 1 \\
-1 & 1 & 0 & 1 & -1 & 0 \\
0 & -1 & 1 & 1 & 0 & -1
\end{array}\right)
$$

which is compatible with (C.9) and the charges (4.8).

Therefore, there is only one backbone structure for $3 \mathrm{HDM}$ with symmetries $\mathbb{Z}_{4}$ or $\mathbb{Z}_{2} \times \mathbb{Z}_{2}$. To obtain the backbone structure (4.7), we just collect the nonequivalent rows of the matrices in (C.9) and (C.12). Analogously, the backbone structure (4.9) is extracted from (C.10).

\section{Height of $\mathrm{p}_{i}^{\prime}$ vectors for maximal $D^{Y}$}

We start from the requirement for maximal $G_{F}$ :

$$
E\left(\mathbf{p}_{3}^{\prime}, \mathbf{p}_{4}^{\prime}, \cdots, \mathbf{p}_{n}^{\prime}\right)= \pm\left|G_{q}\right|_{\text {bound }} .
$$

The determinant-like $E$-function was defined in ref. [15] and coincides with the determinant of $D^{Y}$ after eliminating the first and last columns. When $D^{Y}$ is in the form (3.7), we conclude that only the rows corresponding to $\mathbf{p}_{i}^{\prime}$ contributes non-unit factors. The properties of the $E$-function allows us to expand it using eqs. (5.16) and (5.17) as

$$
\begin{aligned}
& E\left(\mathbf{p}_{3}^{\prime}, \mathbf{p}_{4}^{\prime}, \cdots, \mathbf{p}_{n}^{\prime}\right) \\
& \quad=E\left(\mathbf{p}_{3}, \mathbf{p}_{4}, \cdots, \mathbf{p}_{n}\right)+E\left(-\mathbf{p}_{1}, \mathbf{p}_{4}, \cdots, \mathbf{p}_{n}\right)+E\left(-\mathbf{p}_{2}, \mathbf{p}_{4}, \cdots, \mathbf{p}_{n}\right) \\
& \quad+\text { combinations of }\left[\left(\mathbf{p}_{3 k+1} \rightarrow-\mathbf{p}_{1}\right) \text { or }\left(\mathbf{p}_{3 k+2} \rightarrow-\mathbf{p}_{2}\right) \text { or }\left(\mathbf{p}_{3 k} \rightarrow-\mathbf{p}_{3}\right)\right] .
\end{aligned}
$$


We know that each term in the expansion is either 0 or \pm 1 for height- 2 vectors and so we can write

$$
\mid E(\{n \text { height-2 vectors }\}) \mid \leq 1,
$$

where height-2 vectors denote only $\mathbf{p}$-vectors in this case. To obtain a maximal value, all terms in the expansion should be \pm 1 and no cancellations should occur. In this case, the maximal value (3.3) is attained.

Using the maximality of the expansion in (D.2), we can prove that $\mathbf{p}_{i}^{\prime}, i \geq 4$, are always height-4 vectors. As an example, we calculate

$$
E\left(\mathbf{p}_{3}, \mathbf{p}_{4}^{\prime}, \mathbf{p}_{5}, \cdots, \mathbf{p}_{n}\right)=E\left(\mathbf{p}_{3}, \mathbf{p}_{4}, \mathbf{p}_{5}, \cdots, \mathbf{p}_{n}\right)+E\left(\mathbf{p}_{3},-\mathbf{p}_{1}, \mathbf{p}_{5}, \cdots, \mathbf{p}_{n}\right),
$$

where only $\mathbf{p}_{4}^{\prime}$ is given by (5.16). The two terms in the expansion of (D.4) should add to \pm 2 since they are also present in the expansion (D.2) which should be maximal. Condition (D.3) tells us that $\mathbf{p}_{4}^{\prime}$ should have height $h\left(\mathbf{p}_{4}^{\prime}\right)>2$ but its expansion $\mathbf{p}_{4}^{\prime}=\mathbf{p}_{4}-\mathbf{p}_{1}$, a combination of two p-vectors, implies it has height 4 . Analogous arguments show that all $\mathbf{p}_{i}^{\prime}, i \geq 4$, are height- 4 vectors. We also arrive at the general rule

$$
\mid E \text { (height-4 vector, }\{n-1 \text { height-2 vectors }\}) \mid \leq 2,
$$

where a height-4 vector is restricted to a vector that can be written as a sum of two pvectors. Note that the denomination "sum of two p-vectors" has less meaning than its height since the sum of two $\mathbf{p}$-vectors (height-2) can still be a $\mathbf{p}$-vector (height-2) as

$$
(1,-1,0, \cdots)+(0,1,-1,0, \cdots)=(1,0,-1,0, \cdots) .
$$

For $\mathbf{p}_{3}^{\prime}$, we calculate

$$
\begin{aligned}
E\left(\mathbf{p}_{3}^{\prime}, \mathbf{p}_{4}, \mathbf{p}_{5}, \cdots, \mathbf{p}_{n}\right)= & E\left(\mathbf{p}_{3}, \mathbf{p}_{4}, \mathbf{p}_{5}, \cdots, \mathbf{p}_{n}\right) \\
& +E\left(-\mathbf{p}_{1},-\mathbf{p}_{1}, \mathbf{p}_{5}, \cdots, \mathbf{p}_{n}\right)+E\left(-\mathbf{p}_{2}, \mathbf{p}_{4}, \mathbf{p}_{5}, \cdots, \mathbf{p}_{n}\right),
\end{aligned}
$$

and confirm they also appear in (D.2). Maximality of (D.2), (D.3) and the form of $\mathbf{p}_{3}^{\prime}=$ $\mathbf{p}_{3}-\mathbf{p}_{1}-\mathbf{p}_{2}$ implies $\mathbf{p}_{3}^{\prime}$ is a height-6 vector.

\section{E Height of $\mathrm{p}_{3}^{\prime}$ can not be reduced}

Here we prove that, for maximal $D^{Y}$, row operations on $\left\{\mathbf{p}_{i}^{\prime}\right\}$ in eq. (3.7) cannot transform the whole set into a generic set $\left\{\mathbf{r}_{i}\right\}$ in eq. (3.5), which is composed of rows of at most height 4 . We make repeated use of

Proposition. For vectors $\mathbf{p}_{i}$, of height 2 for which $E\left(\left\{\mathbf{p}_{i}\right\}\right)= \pm 1$, and $\mathbf{u}$, an integer linear combination of $\mathbf{p}_{i}$, if $\left|E\left(\mathbf{p}_{1}, \cdots, \mathbf{u}, \cdots, \mathbf{p}_{n}\right)\right| \geq k$, then $\mathbf{u}$ has height $h(\mathbf{u}) \geq 2 k$.

We prove the equivalent statement: for vectors $\mathbf{p}_{i}$, of height 2 for which $E\left(\left\{\mathbf{p}_{i}\right\}\right)=$ \pm 1 , and $\mathbf{u}$, an integer linear combination of $\mathbf{p}_{i}$, if $\mathbf{u}$ has height $h(\mathbf{u})<2 k$, then $\left|E\left(\mathbf{p}_{1}, \cdots, \mathbf{u}, \cdots, \mathbf{p}_{n}\right)\right|<k$. This statement is the generalization of the rules (D.5) and (D.3), without the equality option. The equality can be included because the height of linear combinations of $\mathbf{p}$-vectors only increases in steps of two. 
The proof follows by induction. The case $k=2$ is evident in (D.3). Now we assume the statement is valid for a vector $\mathbf{u}^{\prime}$ of height $h\left(\mathbf{u}^{\prime}\right)<2(k-1)$. The statement for a vector $\mathbf{u}=\mathbf{u}^{\prime}+\mathbf{p}$, of height $h(\mathbf{u})<2 k$, is true because

$$
\begin{aligned}
\left|E\left(\mathbf{p}_{1}, \cdots, \mathbf{u}, \cdots, \mathbf{p}_{n}\right)\right| & =\left|E\left(\mathbf{p}_{1}, \cdots, \mathbf{u}^{\prime}+\mathbf{p}, \cdots, \mathbf{p}_{n}\right)\right| \\
& \leq\left|E\left(\mathbf{p}_{1}, \cdots, \mathbf{u}^{\prime}, \cdots, \mathbf{p}_{n}\right)\right|+\left|E\left(\mathbf{p}_{1}, \cdots, \mathbf{p}, \cdots, \mathbf{p}_{n}\right)\right| \\
& <(k-1)+1=k .
\end{aligned}
$$

The vector $\mathbf{p}$ is any $\mathbf{p}$-vector of height 2 .

Now, take $\mathbf{p}_{3}^{\prime \prime}=\mathbf{p}_{3}^{\prime}-\mathbf{p}_{4}^{\prime}=\mathbf{p}_{3}-\mathbf{p}_{2}-\mathbf{p}_{4}$ and calculate

$$
\begin{aligned}
E\left(\mathbf{p}_{3}^{\prime \prime},-\mathbf{p}_{1}, \mathbf{p}_{5}, \cdots, \mathbf{p}_{n}\right)=E\left(\mathbf{p}_{3},-\right. & \left.-\mathbf{p}_{1}, \mathbf{p}_{5}, \cdots, \mathbf{p}_{n}\right)+E\left(-\mathbf{p}_{2},-\mathbf{p}_{1}, \mathbf{p}_{5}, \cdots, \mathbf{p}_{n}\right) \\
& +E\left(-\mathbf{p}_{4},-\mathbf{p}_{1}, \mathbf{p}_{5}, \cdots, \mathbf{p}_{n}\right) \\
=E\left(\mathbf{p}_{3},-\right. & \left.-\mathbf{p}_{1}, \mathbf{p}_{5}, \cdots, \mathbf{p}_{n}\right)+E\left(-\mathbf{p}_{2},-\mathbf{p}_{1}, \mathbf{p}_{5}, \cdots, \mathbf{p}_{n}\right) \\
& \quad+E\left(-\mathbf{p}_{1}, \mathbf{p}_{4}, \mathbf{p}_{5}, \cdots, \mathbf{p}_{n}\right) \\
= \pm 3, &
\end{aligned}
$$

for maximal $D^{Y}$. The proposition tell us that $\mathbf{p}_{3}^{\prime \prime}$ has at least height 6 . Changing the sign, $\mathbf{p}_{3}^{\prime \prime}=\mathbf{p}_{3}^{\prime}+\mathbf{p}_{4}^{\prime}$ make things worse and we can conclude that $\mathbf{p}_{3}^{\prime \prime}$ have even greater height. Next, we note that all $\mathbf{p}_{2 k+1}$ are all interchangeable to $\mathbf{p}_{4}$ and the same conclusion follows for $\mathbf{p}_{3}^{\prime \prime}=\mathbf{p}_{3}^{\prime} \mp \mathbf{p}_{3 k+1}$. Generalization to all $\mathbf{p}_{3}^{\prime \prime}=\mathbf{p}_{3}^{\prime} \mp \mathbf{p}_{3 k+2}$ or $\mathbf{p}_{3}^{\prime \prime}=\mathbf{p}_{3}^{\prime} \pm \mathbf{p}_{3 k}$ follows analogously. For the latter, we can calculate $E\left(\mathbf{p}_{3}^{\prime}+\mathbf{p}_{6}^{\prime}, \mathbf{p}_{4}, \mathbf{p}_{5},-\mathbf{p}_{3}, \cdots, \mathbf{p}_{n}\right)$. Since any row operation on the row $\mathbf{p}_{3}^{\prime}$ in (3.8) is generated by one of the previous replacements $\mathbf{p}_{3}^{\prime} \rightarrow \mathbf{p}_{3}^{\prime \prime}$, we arrive at our desired conclusion: we can not decrease the height of $\mathbf{p}_{3}^{\prime}$ by using row operations on $D^{Y}$. Therefore, the equivalence (3.8) is not possible for maximal $D^{Y}$.

\section{F Examples of full models and textures}

By using the methods of section 6, we can construct full models, including the explicit Yukawa terms which gives rise to textures in the quark mass matrices. We show here some examples to illustrate that $D_{\text {full }}^{Y}$ is not univocally constructed from $D^{Y}$. This is a general feature when we try to extend a symmetry from a subsector of a theory to a larger sector involving more fields. In particular, this caveat applies to the methods of sections 6.3 and 6.4 .

The explicit example of section 6.4 constructed the matrix $D_{\text {full }}^{Y}$, shown in (6.9), from the backbone structure in eq. (4.7). The procedure was illustrated in eq. (6.6), where the rows $(1,2,3,4,1,2)$ of $D^{Y}$ were associated to the righthanded quarks $\left(d_{1 R}, d_{2 R}, d_{3 R}, u_{1 R}, u_{2 R}, u_{3 R}\right)$, in this order. If we extract the $\mathbb{Z}_{4}$ charge from $D_{\text {full }}^{Y}$ we obtain

$$
\mathbf{s}=(-1,1,0 ; 1,-1,0 ; 0,2,-1 ;-1,0,2) \text {. }
$$

By using the charge vector, we can obtain all the Yukawa interactions which can be collectively summarized by

$$
\bar{Q}_{i L} d_{j R} \sim\left(\begin{array}{ccc}
v_{2} & v_{1} & 0 \\
v_{1} & v_{2} & v_{3} \\
v_{3} & 0 & v_{2}
\end{array}\right), \quad \bar{Q}_{i L} u_{j R} \sim\left(\begin{array}{ccc}
0 & v_{1} & v_{2} \\
v_{3} & v_{2} & v_{1} \\
v_{1} & v_{3} & 0
\end{array}\right) .
$$


The presence of $v_{3}=\left\langle\phi_{3}^{(0)}\right\rangle$ in the (23) entry of the first matrix denotes the coupling of $\phi_{3}$ with $\bar{Q}_{2 L} d_{3 R}$; for up quark terms, $\tilde{\phi}_{i}$ should be considered. The two matrices also give the order of magnitude of respective quark mass matrices. Note that the terms corresponding to the (31) and (32) entries in the first and second matrices, respectively, are not present in (6.9). These are the missing terms that ensures the $\mathbb{Z}_{4}$ symmetry in both d- and u-sectors separately. We can see that this $\mathbb{Z}_{4}$ symmetry gives rise to two-zero textures [44].

As a second example, we can construct another $\mathbb{Z}_{4}-3 \mathrm{HDM}$ from the same backbone (4.7). In this case, we consider rows $(1,2,3,4,5,6)$ of $D^{Y}$ and associate to righthanded quarks $\left(d_{1 R}, d_{2 R}, d_{3 R}, u_{1 R}, u_{2 R}, u_{3 R}\right)$, in the same order. The matrix $D_{\text {full }}^{Y}$ has 12 rows that can be separated in two submatrices of 6 rows, one for the d-sector and another for the u-sector. Each submatrix leads us to conclude that the d-sector has apparent $\mathbb{Z}_{2} \times \mathrm{U}(1)$ symmetry and the $\mathrm{u}$-sector exhibits apparent $[\mathrm{U}(1)]^{2}$ symmetry. The $\mathbb{Z}_{4}$ charge obtained from $D_{\text {full }}^{Y}$,

$$
\mathbf{s}=(1,-1,0 ;-1,1,0 ; 0,2,1 ; 1,-1,0),
$$

can be used to find one additional term for each d- and u-sectors. The complete Yukawa terms can be summarized in

$$
\bar{Q}_{i L} d_{j R} \sim\left(\begin{array}{ccc}
v_{2} & v_{1} & 0 \\
v_{1} & v_{2} & v_{3} \\
v_{3} & 0 & v_{2}
\end{array}\right), \bar{Q}_{i L} u_{j R} \sim\left(\begin{array}{ccc}
0 & v_{3} & v_{1} \\
v_{3} & 0 & v_{2} \\
v_{1} & v_{2} & v_{3}
\end{array}\right)
$$

The terms that are initially missing correspond to the (31) and (23) entries in the first and second matrices, respectively. With the addition of these terms, we find a $\mathbb{Z}_{4}$ symmetry in the d-sector and an accidental $\mathrm{U}(1)$ symmetry in the u-sector. The presence of an accidental symmetry signals that this second model is essentially different from the first. The symmetries $\mathbb{Z}_{4}$ and U(1) can be checked by using the common charge (F.3). One can further see that the textures in eqs. (F.2) and (F.4) are still general and can accommodate the experimental values for quark masses and mixing parameters since these texture-zeros can be achieved through weak basis change and they impose no physical restriction $[45,46]$.

Open Access. This article is distributed under the terms of the Creative Commons Attribution License (CC-BY 4.0), which permits any use, distribution and reproduction in any medium, provided the original author(s) and source are credited.

\section{References}

[1] ATLAS collaboration, Observation of a new particle in the search for the standard model Higgs boson with the ATLAS detector at the LHC, Phys. Lett. B 716 (2012) 1 [arXiv: 1207.7214] [INSPIRE].

[2] CMS collaboration, Observation of a new boson at a mass of $125 \mathrm{GeV}$ with the CMS experiment at the LHC, Phys. Lett. B 716 (2012) 30 [arXiv:1207.7235] [INSPIRE].

[3] R.N. Mohapatra, From old symmetries to new symmetries: quarks, leptons and B-L, Int. J. Mod. Phys. A 29 (2014) 1430066 [arXiv:1409.7557] [inSPIRE]. 
[4] G. Altarelli and F. Feruglio, Discrete flavor symmetries and models of neutrino mixing, Rev. Mod. Phys. 82 (2010) 2701 [arXiv: 1002.0211] [INSPIRE].

[5] H. Ishimori et al., Non-abelian discrete symmetries in particle physics, Prog. Theor. Phys. Suppl. 183 (2010) 1 [arXiv:1003.3552] [INSPIRE].

[6] S.F. King and C. Luhn, Neutrino mass and mixing with discrete symmetry, Rept. Prog. Phys. 76 (2013) 056201 [arXiv:1301.1340] [InSPIRE].

[7] S. Morisi and J.W.F. Valle, Neutrino masses and mixing: a flavour symmetry roadmap, Fortsch. Phys. 61 (2013) 466 [arXiv:1206.6678] [INSPIRE].

[8] A.Y. Smirnov, Neutrino mass, mixing and discrete symmetries, J. Phys. Conf. Ser. 447 (2013) 012004 [arXiv:1305.4827] [InSPIRE].

[9] G.R. Farrar and P. Fayet, Phenomenology of the production, decay and detection of new hadronic states associated with supersymmetry, Phys. Lett. B 76 (1978) 575 [INSPIRE].

[10] S. Dimopoulos, S. Raby and F. Wilczek, Proton decay in supersymmetric models, Phys. Lett. B 112 (1982) 133 [INSPIRE].

[11] B. Petersen, M. Ratz and R. Schieren, Patterns of remnant discrete symmetries, JHEP 08 (2009) 111 [arXiv:0907.4049] [INSPIRE].

[12] R. Schieren, Discrete symmetries in the MSSM, arXiv:1102.4611 [INSPIRE].

[13] M.-C. Chen, M. Ratz and V. Takhistov, $R$ parity violation from discrete $R$ symmetries, Nucl. Phys. B 891 (2015) 322 [arXiv:1410.3474] [INSPIRE].

[14] R. González Felipe and H. Serôdio, Abelian realization of phenomenological two-zero neutrino textures, Nucl. Phys. B 886 (2014) 75 [arXiv:1405.4263] [INSPIRE].

[15] I.P. Ivanov and C.C. Nishi, Abelian symmetries of the N-Higgs-doublet model with Yukawa interactions, JHEP 11 (2013) 069 [arXiv:1309.3682] [INSPIRE].

[16] T.D. Lee, A theory of spontaneous T violation, Phys. Rev. D 8 (1973) 1226 [InSPIRE].

[17] S. Weinberg, Gauge theory of CP-violation, Phys. Rev. Lett. 37 (1976) 657 [INSPIRE].

[18] G.C. Branco, J.M. Gerard and W. Grimus, Geometrical T violation, Phys. Lett. B 136 (1984) 383 [inSPIRE].

[19] I. de Medeiros Varzielas and D. Emmanuel-Costa, Geometrical CP-violation, Phys. Rev. D 84 (2011) 117901 [arXiv:1106 .5477] [inSPIRE].

[20] I. de Medeiros Varzielas, Geometrical CP-violation in multi-Higgs models, JHEP 08 (2012) 055 [arXiv: 1205.3780] [INSPIRE].

[21] I.P. Ivanov and L. Lavoura, Geometrical CP-violation in the N-Higgs-doublet model, Eur. Phys. J. C 73 (2013) 2416 [arXiv: 1302.3656] [InSPIRE].

[22] S.L. Glashow and S. Weinberg, Natural conservation laws for neutral currents, Phys. Rev. D 15 (1977) 1958 [INSPIRE].

[23] E. Ma, Verifiable radiative seesaw mechanism of neutrino mass and dark matter, Phys. Rev. D 73 (2006) 077301 [hep-ph/0601225] [INSPIRE].

[24] I.P. Ivanov, Two-Higgs-doublet model from the group-theoretic perspective, Phys. Lett. B 632 (2006) 360 [hep-ph/0507132] [INSPIRE].

[25] C.C. Nishi, CP violation conditions in N-Higgs-doublet potentials, Phys. Rev. D 74 (2006) 036003 [Erratum ibid. D 76 (2007) 119901] [hep-ph/0605153] [INSPIRE]. 
[26] M. Maniatis, A. von Manteuffel, O. Nachtmann and F. Nagel, Stability and symmetry breaking in the general two-Higgs-doublet model, Eur. Phys. J. C 48 (2006) 805 [hep-ph/0605184] [INSPIRE].

[27] I.P. Ivanov, Minkowski space structure of the Higgs potential in 2HDM, Phys. Rev. D 75 (2007) 035001 [Erratum ibid. D 76 (2007) 039902] [hep-ph/0609018] [INSPIRE].

[28] I.P. Ivanov, Minkowski space structure of the Higgs potential in 2HDM. II. Minima, symmetries and topology, Phys. Rev. D 77 (2008) 015017 [arXiv:0710.3490] [INSPIRE].

[29] P.M. Ferreira, H.E. Haber, M. Maniatis, O. Nachtmann and J.P. Silva, Geometric picture of generalized-CP and Higgs-family transformations in the two-Higgs-doublet model, Int. J. Mod. Phys. A 26 (2011) 769 [arXiv:1010.0935] [InSPIRE].

[30] P.M. Ferreira and J.P. Silva, Abelian symmetries in the two-Higgs-doublet model with fermions, Phys. Rev. D 83 (2011) 065026 [arXiv:1012.2874] [INSPIRE].

[31] N.G. Deshpande and E. Ma, Pattern of symmetry breaking with two Higgs doublets, Phys. Rev. D 18 (1978) 2574 [inSPIRE].

[32] B. Grzadkowski, M. Maniatis and J. Wudka, The bilinear formalism and the custodial symmetry in the two-Higgs-doublet model, JHEP 11 (2011) 030 [arXiv:1011.5228] [INSPIRE].

[33] C.C. Nishi, Custodial $\mathrm{SO}(4)$ symmetry and CP-violation in N-Higgs-doublet potentials, Phys. Rev. D 83 (2011) 095005 [arXiv:1103.0252] [InSPIRE].

[34] R.A. Battye, G.D. Brawn and A. Pilaftsis, Vacuum topology of the two Higgs doublet model, JHEP 08 (2011) 020 [arXiv:1106.3482] [INSPIRE].

[35] A. Pilaftsis, On the classification of accidental symmetries of the two Higgs doublet model potential, Phys. Lett. B 706 (2012) 465 [arXiv:1109.3787] [INSPIRE].

[36] P.S.B. Dev and A. Pilaftsis, Maximally symmetric two Higgs doublet model with natural standard model alignment, JHEP 12 (2014) 024 [arXiv:1408.3405] [INSPIRE].

[37] I.P. Ivanov and E. Vdovin, Classification of finite reparametrization symmetry groups in the three-Higgs-doublet model, Eur. Phys. J. C 73 (2013) 2309 [arXiv:1210.6553] [INSPIRE].

[38] I.P. Ivanov and C.C. Nishi, Symmetry breaking patterns in 3HDM, JHEP 01 (2015) 021 [arXiv: 1410.6139] [INSPIRE].

[39] I.P. Ivanov, V. Keus and E. Vdovin, Abelian symmetries in multi-Higgs-doublet models, J. Phys. A 45 (2012) 215201 [arXiv:1112.1660] [inSPIRE].

[40] V.A. Solano et al., IntegerSmithNormalForm, Mathematica notebook, Wolfram Library Archive, http://library.wolfram.com/infocenter/MathSource/6621.

[41] M. Leurer, Y. Nir and N. Seiberg, Mass matrix models, Nucl. Phys. B 398 (1993) 319 [hep-ph/9212278] [INSPIRE].

[42] R. González Felipe et al., Constraining multi-Higgs flavour models, Eur. Phys. J. C 74 (2014) 2953 [arXiv:1401.5807] [InSPIRE].

[43] G.C. Branco et al., Theory and phenomenology of two-Higgs-doublet models, Phys. Rept. 516 (2012) 1 [arXiv:1106.0034] [INSPIRE].

[44] W. Grimus, A.S. Joshipura, L. Lavoura and M. Tanimoto, Symmetry realization of texture zeros, Eur. Phys. J. C 36 (2004) 227 [hep-ph/0405016] [INSPIRE]. 
[45] H. Fritzsch and Z.-z. Xing, The light quark sector, CP-violation and the unitarity triangle, Nucl. Phys. B 556 (1999) 49 [hep-ph/9904286] [INSPIRE].

[46] G.C. Branco, D. Emmanuel-Costa and R. Gonzalez Felipe, Texture zeros and weak basis transformations, Phys. Lett. B 477 (2000) 147 [hep-ph/9911418] [INSPIRE]. 